\title{
Tetrad formalism for numerical relativity on conformally compactified constant mean curvature hypersurfaces
}

\author{
James M. Bardeen, ${ }^{1}$ Olivier Sarbach, ${ }^{2}$ and Luisa T. Buchman ${ }^{3}$ \\ ${ }^{1}$ Physics Department, University of Washington, Seattle, Washington 98195 USA \\ ${ }^{2}$ Instituto de Física y Matemáticas, Universidad Michoacana de San Nicolás de Hidalgo, \\ Edificio C-3, Ciudad Universitaria, 58040 Morelia, Michoacán, México \\ ${ }^{3}$ Theoretical Astrophysics, California Institute of Technology, Pasadena, California 91125 USA
}

(Received 28 January 2011; published 24 May 2011)

\begin{abstract}
We present a new evolution system for Einstein's field equations which is based on tetrad fields and conformally compactified hyperboloidal spatial hypersurfaces which reach future null infinity. The boost freedom in the choice of the tetrad is fixed by requiring that its timelike leg be orthogonal to the foliation, which consists of constant mean curvature slices. The rotational freedom in the tetrad is fixed by the 3D Nester gauge. With these conditions, the field equations reduce naturally to a first-order constrained symmetric hyperbolic evolution system which is coupled to elliptic equations for the gauge variables. The conformally rescaled equations are given explicitly, and their regularity at future null infinity is discussed. Our formulation is potentially useful for high accuracy numerical modeling of gravitational radiation emitted by inspiraling and merging black hole binaries and other highly relativistic isolated systems.
\end{abstract}

DOI: 10.1103/PhysRevD.83.104045

PACS numbers: 04.20.Ha, 04.20.-q, 04.25.D-

\section{INTRODUCTION}

The calculation of gravitational waveforms generated by the inspiral and coalescence of binary black holes is now a routine matter for numerical relativists (see Refs. [1,2] for recent reviews). One of the most pressing goals for those working in the field today is to compute these waveforms as accurately as possible, without losing computational efficiency. Optimally, this goal is accomplished by including future null infinity $\left(\mathcal{J}^{+}\right)$in the computational grid because then the Bondi news function [3-5], which contains all the gravitational wave information, can be read off without the need for extrapolation. One way to include $\mathcal{J}^{+}$ in the numerical grid is Cauchy characteristic matching (see Ref. [6] for a review), which joins a standard $3+1$ Cauchy code in an interior region with a characteristic code in an outer region. However, arranging stable data transfer in both directions at the interface between the two regions is difficult in general; hence, a modified approach called Cauchy characteristic extraction (CCE) [6-8], in which data is extracted from the interior of a standalone Cauchy development and extrapolated to $\mathcal{J}^{+}$using a characteristic code, is now often used to extract binary black hole inspiral and merger waveforms at $\mathcal{J}^{+}$. While CCE is certainly a significant improvement over wave extraction at the finite boundary of a conventional Cauchy code, potential sources of error remain. Initial conditions for the characteristic code cannot easily be made compatible with the Cauchy time development. Also, either errors in boundary conditions for the Cauchy code can affect the CCE or there is insufficient time for all the junk radiation present in the initial data of the Cauchy code to escape. Furthermore, the extraction imposes a gauge on the characteristic code in which the expansion of $\mathcal{J}^{+}$generally does not vanish, making it somewhat of a challenge to obtain the Bondi news accurately [8].

An approach which, we argue, has greater potential for high accuracy waveform calculations is to conformally compactify the spatial geometry on hyperboloidal spacelike hypersurfaces, as originally advocated by Friedrich [9], but with elliptic equations determining the gauge degrees of freedom. Hyperboloidal hypersurfaces behave like conventional time slices near the compact object sources, but smoothly transition to asymptotically approach null slices in the physical space-time at $\mathcal{J}^{+}$. For a wide class of problems of physical interest, the compactified conformal geometry is regular, and the entire space section can be represented on a finite resolution and relatively small coordinate grid, enabling (we expect) efficient simulations and accurate waveform calculations. We can choose boundary conditions on the elliptic gauge equations at $\mathcal{J}^{+}$which make the expansion of $\mathcal{J}^{+}$vanish, and thereby simplify reading off the asymptotic gravitational wave amplitudes. No outer boundary conditions are required on the dynamical variables, since $\mathcal{J}^{+}$is an ingoing null hypersurface in the conformally compactified geometry.

Friedrich [9] derived a first-order symmetric fully hyperbolic system evolved on compactified hyperboloidal hypersurfaces, with tetrad components of the Weyl tensor as the fundamental dynamical variables and with the Bianchi identities providing the evolution equations for these variables. The system is manifestly regular at $\mathcal{J}^{+}$, thanks to the conformal invariance of the Weyl tensor. We refer the reader to the review article [10] by Frauendiener for further details, including references to numerical work based on this formulation. The system is very useful for obtaining analytic results on existence and uniqueness of radiative spacetimes (e.g. [11]). 
Metric-based proposals for solving the Einstein equations on conformally compactified hyperboloidal hypersurfaces include the completely hyperbolic generalized harmonic scheme of Zenginoğlu [12] and the mixed hyperbolic-elliptic formulation of Moncrief and Rinne [13]. Both of these are second order in spatial derivatives. Rinne [14] has implemented a simplified version of the Moncrief-Rinne formalism in an axisymmetric finite difference code, and demonstrated long-term stable dynamical evolution of the Einstein equations. Zenginoğlu and Tiglio [15] and Zenginoğlu and Kidder [16] have successfully evolved wave equations for test fields on compactified hyperboloidal hypersurfaces on fixed black hole backgrounds. Conformally flat hyperboloidal Bowen-York binary black hole initial data have been constructed in [17].

The tetrad framework we present in this paper uses the dyadic notation of the Einstein-Bianchi system of Estabrook and Wahlquist [18], modified to allow for conformal rescaling. Instead of basing the dynamical system on the Weyl tensor and the Bianchi identities, our fundamental variables are the 24 tetrad connection coefficients (i.e., Ricci rotation coefficients) evolved using the Einstein equations. In the context of certain dynamical gauge conditions, this system can be put in a first-order symmetric hyperbolic form, the "WEBB" equations [19,20]. The mathematical structure of these equations is analyzed in Refs. [21,22], and 1D numerical tests are presented in Ref. [23]. A more general discussion of symmetric hyperbolic systems for the Einstein equations and their suitability for numerical relativity is given by Friedrich in [24]. Purely hyperbolic numerical evolution in general relativity, particularly in a first-order scheme, needs to accurately preserve a fairly large number of constraints.

Our approach here, like that of Moncrief and Rinne [13], is a mixed hyperbolic-elliptic system based on a special class of hyperboloidal hypersurfaces called constant mean curvature (CMC) hypersurfaces, for which the trace of the extrinsic curvature is uniform in both space and time. Some of the elliptic equations are singular at $\mathcal{J}^{+}$, where the conformal factor vanishes, which forces the solutions to have a highly constrained asymptotic behavior. This is a feature, not a bug, since it enables a detailed analysis of the asymptotic regularity conditions which must be imposed in order to obtain nonsingular evolution of the conformal geometry. We differ from Moncrief and Rinne in that our evolution equations are first order in space as well as time, and include connection coefficients of the conformal spatial geometry in our fundamental set of dynamical variables. Also, instead of the coordinate metric and coordinate components of tensors, our approach is based on orthonormal tetrad basis vectors. The tetrad components of all tensors are coordinate scalars, and the metric for raising and lowering tetrad indices is trivial. Consequently, the resulting equations are simpler in form and in interpretation than those of Moncrief and Rinne.
The extra gauge degrees of freedom in our tetrad formalism corresponding to local Lorentz boosts and rotations of the tetrad frames are fixed in a natural way which simplifies the equations and reduces the number of variables compared to a general tetrad formalism. The timelike vector of the tetrad is chosen to be the unit normal to the CMC hypersurfaces, which constrains the triad of spatial vectors to be tangent to the CMC hypersurfaces. The rotational degrees of freedom in the conformal spatial triad vectors are fixed by imposing the 3D Nester gauge [25]. In a certain global sense, the Nester gauge makes the triad vectors at different points in the hypersurface as nearly related by parallel transport as possible. The Nester gauge leads naturally [26] to a choice of conformal factor, which satisfies an elliptic equation derived from the Hamiltonian constraint and is enforced by elliptic equations for potentials that determine the conformal angular velocity of the triad frames and the trace of the conformal extrinsic curvature. Of the 24 tetrad connection coefficients, only 10 are dynamical and form a Maxwell-like symmetric hyperbolic system. The remaining evolution equations are the nine linear advection equations for the coordinate components of the three spatial triad vectors.

As in Ref. [13], we put considerable effort into analyzing the properties of the equations and their solutions in the neighborhood of $\mathcal{J}^{+}$. The evolution equations for the dynamical variables contain terms which have a conformal factor in the denominator and therefore are potentially singular at $\mathcal{J}^{+}$. Certain well-known regularity conditions must be imposed to make these terms finite, such as the "zero-shear" condition on the null generators of $\mathcal{J}^{+}$(see Ref. [27]). Once imposed in the initial conditions, they are preserved by the evolution equations. An additional and stronger regularity condition is also usually assumed, that the conformally invariant Weyl tensor vanish at $\mathcal{J}^{+}$. We refer to this as the "Penrose regularity condition", and it is also automatically preserved by the evolution equations. The review of Frauendiener [10] puts the various regularity and smoothness assumptions at $\mathcal{J}^{+}$in context.

The question of how much smoothness in the conformal geometry at $\mathcal{J}^{+}$is, in some sense, physically generic has been the matter of considerable debate in the literature. The original analyses of Bondi et al. [3] and Sachs [4] assumed analyticity, but the more systematic conformal analysis of Penrose [28] reduced this to $C^{3}$ smoothness. Later, Chruściel et al. [29] showed that "polyhomogeneous" terms in the expansion of the angular part of the metric in Bondi-Sachs coordinates, terms of the form $x^{n}(\log x)^{m}$, where $x \equiv 1 / r$, are consistent with the Einstein equations and the ability to define a physically suitable Bondi energy. The coefficient of the "leading $\log$ " term is a constant during evolution, so if these polyhomogeneous terms are ever present they were always present back to past null infinity, where they are associated with incoming radiation. 
We conclude from this that in the context of typical astrophysical problems, where one can assume there is no or negligible incoming radiation at past null infinity, it is perfectly appropriate to exclude polyhomogeneous terms of the type contemplated in Ref. [29] from ever being present. Similarly, violations of the regularity conditions described above, including the Penrose regularity condition, can only be present if they were present at past null infinity, and cannot be generated by any dynamics of the physical system. However, as was shown in Ref. [27] and as we derive in Appendix A, polyhomogeneous terms in both the conformal and physical extrinsic curvature and the conformal factor are generically present when the space-time is foliated on CMC hypersurfaces. In fact, we show that they must be present whenever outgoing radiation is present at $\mathcal{J}^{+}$, and are not present otherwise. These terms arise from the special character of the momentum constraint equation and the elliptic equation for the conformal factor on a CMC slice as it becomes asymptotically null at $\mathcal{J}^{+}$. In a more flexible hyperboloidal slicing, where the trace of the extrinsic curvature only approaches a constant at $\mathcal{J}^{+}$, the polyhomogeous terms need not be present. In fact, they are clearly not present when the space-time is evolved using the regular conformal field equations of Friedrich [9], which allows the foliation to evolve as part of the hyperbolic system.

There are some significant advantages to our mixed hyperbolic-elliptic system. The boundary conditions on the gauge variables determined by the elliptic equations can be adjusted to simplify and control the geometric properties of and coordinates on $\mathcal{J}^{+}$. For instance, the boundary condition on the elliptic equation for the conformal lapse, together with the boundary condition on the trace of the conformal extrinsic curvature, can keep the intrinsic conformal geometry of the intersection of the CMC hypersurface with $\mathcal{J}^{+}$that of a two-sphere of constant area and the time coordinate on the CMC hypersurfaces equal to the retarded Minkowski time at $\mathcal{J}^{+}$. The shift vector controls the evolution of the spatial coordinates. We propose a few alternative vector elliptic equations for the shift, all with a boundary condition chosen to keep $\mathcal{J}^{+}$at a fixed coordinate radius.

The final conformally rescaled system is a symmetric hyperbolic evolution scheme which is coupled to an elliptic system for the conformal factor and the gauge variables. Hyperbolic-elliptic formulations have been discussed in the literature, both from mathematical and numerical viewpoints. A hyperbolic-elliptic formulation of Einstein's equations in spatial harmonic coordinates on CMC hypersurfaces, very similar to the Moncrief-Rinne formulation but without the conformal compactification, has been proven well-posed by Andersson and Moncrief [30]. Note that the Andersson-Moncrief formulation is not constrained-elliptic equations are solved only for the lapse and shift. The Meudon group proposed a fully constrained, conformally rescaled system [31] for which both numerical tests and mathematical analyses have been performed [31-33]. Finally, elliptic equations have been periodically enforced in a constraint projection scheme [34] for a firstorder reduction of a scalar field equation on a black hole background, and shown not to be prohibitively expensive. Therefore, we hope that a numerical implementation of the proposal presented in this paper with efficient elliptic solvers such as those in the Spectral Einstein Code SPEC $[35,36]$ will be possible with reasonable computational effort, especially since we would need relatively small grid sizes due to conformal compactification and the relatively small number of wave oscillations out to $\mathcal{J}^{+}$on a CMC hypersurface.

Our paper is organized as follows. In Sec. II, we specify our choice of variables and notation and, based on the hypersurface-orthogonal gauge, we present the $3+1$ decomposition of the triad and connection coefficients as originally developed in the dyadic formalism of Ref. [18]. In Sec. III, we discuss the evolution and constraint equations of the WEBB tetrad formulation when specialized to a hypersurface-orthogonal gauge, and we decompose both the extrinsic curvature and the dyadic form of the spatial connection coefficients into symmetric trace-free, antisymmetric, and trace parts. The antisymmetric and trace parts are fixed by our gauge conditions which are discussed in Sec. IV. While the antisymmetric part of the extrinsic curvature is identically zero, its trace is fixed to a constant by the CMC slicing condition, and the trace and antisymmetric parts of the dyadic form of the spatial connection coefficients are determined by the 3D Nester gauge condition (the latter up to a gradient). The remaining symmetric trace-free parts are governed by a nice Maxwell-like symmetric hyperbolic evolution system which is coupled to advection equations for the triad vectors and elliptic equations for the gauge variables. The conformal decomposition is performed in Sec. V. The Nester gauge is covariant with respect to conformal transformations, and this property motivates a natural choice for the conformal factor. With this choice, the antisymmetric part of the dyadic representation of the conformal connection coefficients is zero, which further simplifies the equations. Also in Sec. V, we derive manifestly regular elliptic equations guaranteeing that these gauge conditions are preserved throughout evolution, and we discuss possible elliptic equations for the shift. In Sec. VI, we analyze necessary conditions to impose on the fields at $\mathcal{J}^{+}$in order to have a regular time evolution. Furthermore, we evaluate explicitly the apparently singular terms at $\mathcal{J}^{+}$in the right-hand side of the evolution equations. Our evolution system, together with its constraints and elliptic equations for the gauge variables including boundary conditions, is summarized in Sec. VII, and issues such as comparisons with other approaches and extraction of gravitational waveforms are discussed in Sec. VIII. 
More technical aspects relevant to our formulation are given in Appendices $\mathrm{A}, \mathrm{B}$, and $\mathrm{C}$ where we discuss, respectively, asymptotic expansions of the fields near $\mathcal{J}^{+}$, the constraint propagation system, and the relation of the 3D Nester gauge condition to the 3D Dirac equation.

Throughout this paper, we use the sign convention $(-,+,+,+)$ for the metric. We denote by $\dot{\mathcal{J}}^{+}$the $2 \mathrm{D}$ intersection of the $\mathcal{J}^{+}$null hypersurface with a hyperboloidal CMC spatial hypersurface, and by the $\doteq$ sign an equality that holds only at $\mathcal{J}^{+}$. We adopt the Wald [37] sign convention for the extrinsic curvature of a spatial hypersurface, so it is positive for expansion toward the future.

\section{3 + 1 DECOMPOSITION}

The tetrads in the spinor formulation of Newman and Penrose [38] are aligned along null congruences and are therefore conducive to understanding radiative properties of a space-time. In contrast, in the tetrad formulation presented by Estabrook and Wahlquist [18], the tetrad is oriented with respect to a preferred timelike congruence. This can be useful if a physically preferred timelike direction, such as a fluid 4-velocity, exists. However, this is not the case in vacuum. Our choice is to orient the timelike leg of the tetrad orthogonal to the CMC hypersurfaces of constant coordinate time $\Sigma_{t}$. It follows then that the spacelike legs of the tetrad are tangent to $\Sigma_{t}$. This is called a hypersurface-orthogonal gauge condition, and we assume this gauge condition throughout the paper.

Our notation follows that of Ref. [19]. Space-time indices $(0-3)$ are Greek and spatial indices (1-3) are Latin. The beginning of the alphabet is used for tetrad indices, and the middle of the alphabet is used for coordinate indices. Spatial tetrad indices will not be raised and lowered, since the spatial metric in the tetrad basis is trivial. In Ref. [19], there was a distinction between the tetrad vectors with components $\lambda_{\alpha}{ }^{\mu}$ and the spatial triad vectors $\mathbf{B}_{a}$ defined as projections of the spatial tetrad vectors tangent to $\Sigma_{t}$, with only spatial components $B_{a}{ }^{k}$. In a hypersurface-orthogonal gauge, the spatial tetrad vectors have zero time components and are identical to the $\mathbf{B}_{a}$. The directional derivatives along the three spatial legs of the tetrad are then ${ }^{1}$

$$
D_{a}=B_{a}{ }^{k} \frac{\partial}{\partial x^{k}} .
$$

The Lie derivative along the timelike leg of the tetrad is written in terms of the lapse function $(\alpha)$ and the shift vector $\left(\beta^{k}\right)$ :

$$
D_{0}=\frac{1}{\alpha}\left(\frac{\partial}{\partial t}-\mathcal{L}_{\beta}\right)
$$

\footnotetext{
${ }^{1}$ The vector $A_{a}$ of Ref. [19], which is the velocity of the tetrad congruence relative to a hypersurface rest frame, is identically zero.
}

Let us stress that we regard variables with pure tetrad indices (such as $K_{a b}, N_{a b}, a_{b}$ and $\omega_{b}$ below) as scalar fields on $\Sigma_{t}$. In this sense, the Lie derivative operator $\mathcal{L}_{\beta}$ with respect to the shift acts as the directional derivative on such variables. In contrast to this, we have $\alpha D_{0} B_{a}{ }^{i}=$ $\left(\partial_{t}-\beta^{j} \partial_{j}\right) B_{a}{ }^{i}+D_{a} \beta^{i}$ which differs from the definition in [19], where $\alpha D_{0} B_{a}{ }^{i}=\left(\partial_{t}-\beta^{j} \partial_{j}\right) B_{a}{ }^{i}$.

The coordinate basis space-time metric $g_{\mu \nu}$ and the spatial metric $h_{i j}$ can be calculated from the tetrad and triad vectors as

$$
g^{\mu \nu}=\eta^{\alpha \beta} \lambda_{\alpha}{ }^{\mu} \lambda_{\beta}{ }^{\nu}, \quad h^{i j}=B_{a}{ }^{i} B_{a}{ }^{j},
$$

where $\eta^{\alpha \beta}$ is the Minkowski metric. The connection coefficients with respect to the Levi-Civita connection $\nabla$ of $g_{\mu \nu}$ in the tetrad basis are calculated from the commutators,

$$
\begin{aligned}
\Gamma_{\alpha \beta \gamma} & =\lambda_{\alpha} \cdot \nabla_{\gamma} \lambda_{\beta}=-\Gamma_{\beta \alpha \gamma} \\
& =\frac{1}{2}\left(\lambda_{\beta} \cdot\left[\lambda_{\alpha}, \lambda_{\gamma}\right]+\lambda_{\gamma} \cdot\left[\lambda_{\alpha}, \lambda_{\beta}\right]-\lambda_{\alpha} \cdot\left[\lambda_{\beta}, \lambda_{\gamma}\right]\right) .
\end{aligned}
$$

In the dyadic formalism [18], the 24 independent $\Gamma_{\alpha \beta \gamma}$ are decomposed into two $3 \times 3$ matrices

$$
K_{a b} \equiv \Gamma_{b 0 a}, \quad N_{a b} \equiv \frac{1}{2} \varepsilon_{b c d} \Gamma_{c d a},
$$

and two vectors

$$
a_{b} \equiv \Gamma_{b 00}, \quad \omega_{b} \equiv-\frac{1}{2} \varepsilon_{b c d} \Gamma_{c d 0} .
$$

In the hypersurface-orthogonal gauge, the $K_{a b}$ are the triad components of the symmetric extrinsic curvature tensor of $\Sigma_{t}$. The sign of $K_{a b}$ is opposite to that of Misner, Thorne, and Wheeler [39], in that positive values imply expansion of the normals, not contraction. The $N_{a b}$ describe the induced connection coefficients of $\Sigma_{t}$. It is often convenient to write the antisymmetric part of $N_{a b}$ as the vector

$$
n_{b} \equiv \frac{1}{2} \varepsilon_{b c d} N_{c d}
$$

The vectors $a_{b}$ and $\omega_{b}$ are, respectively, the acceleration and the angular velocity relative to Fermi-Walker transport of the tetrad frame. The acceleration vector field is given by the gradient of the logarithm of the lapse,

$$
a_{b}=D_{b}(\log \alpha) \text {. }
$$

\section{BASIC EQUATIONS}

Here we write the basic Einstein evolution and constraint equations of the WEBB tetrad formulation (these are derived in Ref. [19] from the Einstein equations and the Riemann constraints, see also Appendix B), specialized to a hypersurface-orthogonal gauge. Note that $K_{a b}$ is explicitly symmetric. The resulting evolution equations for $K_{a b}$ and $N_{a b}$, respectively, are 


$$
\begin{aligned}
& D_{0} K_{a b}-\varepsilon_{a c d} D_{c} N_{d b} \\
& =\left(D_{a}+a_{a}\right) a_{b}-\varepsilon_{b c d} N_{a c} a_{d}+2 \varepsilon_{c d(a} K_{b) c} \omega_{d}+N N_{a b} \\
& +\frac{1}{2} \varepsilon_{a d f} \varepsilon_{b c e}\left(K_{d c} K_{f e}-N_{d c} N_{f e}\right)-K_{a c} K_{c b}-N_{c a} N_{c b} \\
& +8 \pi G\left[\sigma_{a b}-\frac{1}{2} \delta_{a b}\left(\sigma_{c c}+\rho\right)\right] \\
& \quad D_{0} N_{a b}+\varepsilon_{a c d} D_{c} K_{d b} \\
& =-\left(D_{a}+a_{a}\right) \omega_{b}+\varepsilon_{b c d}\left(K_{a c} a_{d}+N_{a c} \omega_{d}\right) \\
& \quad+\varepsilon_{a c d} N_{c b} \omega_{d}-N K_{a b}+2 N_{c[a} K_{b] c} \\
& \quad+\varepsilon_{a d f} \varepsilon_{b c e} N_{d c} K_{f e}-8 \pi G \varepsilon_{a b c} j_{c}
\end{aligned}
$$

where $N$ is the trace of $N_{a b}, K$ is the trace of $K_{a b}$, and $a_{b}=$ $D_{b}(\log \alpha)$. Here, we have also defined the energy density $\rho=\tau_{00}$, the energy flux $j_{b}=-\tau_{0 b}$, and the stress tensor $\sigma_{a b}=\tau_{a b}$, where $\tau_{\mu \nu}$ denotes the stress-energy tensor.

The Hamiltonian and momentum constraint equations are, respectively,

$$
\begin{aligned}
2 D_{a} n_{a}= & N_{a b} N_{a b}+\frac{1}{2}\left(K_{a b} K_{a b}-N_{a b} N_{b a}-K^{2}-N^{2}\right) \\
& +8 \pi G \rho,
\end{aligned}
$$

$D_{b} K_{a b}-D_{a} K=-\varepsilon_{a b c} K_{b d} N_{d c}+2 K_{a c} n_{c}-8 \pi G j_{a}$.

There is also a constraint for $N_{a b}$ analogous to the momentum constraint equation, which is obtained from the Riemann identities:

$$
D_{b} N_{a b}-D_{a} N=-\varepsilon_{a b c} N_{b d} N_{d c} .
$$

Evolution and constraint equations for the spatial triad vectors $\mathbf{B}_{\mathbf{a}}$ are derived from the commutators of the orthonormal basis vectors and are

$$
\begin{gathered}
D_{0} B_{a}{ }^{k}=-\varepsilon_{a b c} \omega_{b} B_{c}{ }^{k}-K_{a c} B_{c}{ }^{k}, \\
\varepsilon_{a b c} D_{b} B_{c}{ }^{k}=N_{b a} B_{b}{ }^{k}-N B_{a}{ }^{k} .
\end{gathered}
$$

In the following, it will be useful to separate out the symmetric, trace-free parts of $K_{a b}$ and $N_{a b}$, denoted by $\hat{K}_{a b}$ and $\hat{N}_{a b}$, so that

$$
K_{a b}=\hat{K}_{a b}+\frac{\delta_{a b}}{3} K, \quad N_{a b}=\hat{N}_{a b}+\varepsilon_{a b c} n_{c}+\frac{\delta_{a b}}{3} N .
$$

From Eqs. (9) and (10), we obtain the following evolution equations for $\hat{K}_{a b}$ and $\hat{N}_{a b}$ :

$$
\begin{aligned}
D_{0} \hat{K}_{a b}-D_{c} \hat{N}_{d(a} \varepsilon_{b) c d} \\
=\left\{\left(D_{(a}+a_{(a}\right) a_{b)}-\left(D_{(a}-a_{(a}\right) n_{b)}\right. \\
\quad-K \hat{K}_{a b}+\frac{2 N}{3} \hat{N}_{a b}-2 \hat{N}_{a c} \hat{N}_{c b} \\
\left.+\varepsilon_{c d(a}\left[\hat{N}_{b) c}\left(2 n_{d}-a_{d}\right)+2 \hat{K}_{b) c} \omega_{d}\right]+8 \pi G \sigma_{a b}\right\}^{T F} \\
D_{0} \hat{N}_{a b}+D_{c} \hat{K}_{d(a} \varepsilon_{b) c d} \\
=\left\{-\left(D_{(a}+a_{(a}\right) \omega_{b)}-\frac{4 N}{3} \hat{K}_{a b}-\frac{K}{3} \hat{N}_{a b}\right. \\
\left.\quad+2 \hat{K}_{c(a} \hat{N}_{b) c}+\varepsilon_{c d(a}\left[\hat{K}_{b) c} a_{d}+2 \hat{N}_{b) c} \omega_{d}\right]\right\}^{T F}
\end{aligned}
$$

where $\{\cdots\}^{T F}$ denotes the trace-free part of the expression inside the parenthesis. The evolution equation for the trace of the extrinsic curvature, from which an elliptic equation for the lapse will be derived in Sec. IVA, is

$$
\begin{aligned}
D_{0} K= & \left(D_{b}+a_{b}-2 n_{b}\right) a_{b}-\hat{K}_{a b} \hat{K}_{a b}-\frac{1}{3} K^{2} \\
& -4 \pi G\left(\sigma_{a a}+\rho\right) .
\end{aligned}
$$

We have used the Hamiltonian constraint, Eq. (11), to eliminate the scalar 3-curvature. The evolution equations for the trace and the antisymmetric part of $N_{a b}$, from which we will derive elliptic equations for $\omega_{a}$ and the time derivative of the conformal factor in Sec. VA, are

$$
\begin{aligned}
D_{0} N= & -\left(D_{a}+a_{a}\right) \omega_{a}-\hat{N}_{a b} \hat{K}_{a b}-\frac{1}{3} K N, \\
2 D_{0} n_{a}= & -\varepsilon_{a b c}\left(D_{b}+a_{b}-2 n_{b}\right) \omega_{c}-\frac{2 K}{3}\left(a_{a}+n_{a}\right) \\
& -\varepsilon_{a b c} \hat{K}_{b d} \hat{N}_{d c}+\hat{K}_{a b}\left(a_{b}+n_{b}\right)-8 \pi G j_{a} .
\end{aligned}
$$

The second equation has been simplified by applying the momentum constraint Eq. (12).

The Hamiltonian and momentum constraint Eqs. (11) and (12), expressed in terms of the symmetric trace-free and antisymmetric parts of $K_{a b}$ and $N_{a b}$, are

$$
\begin{aligned}
D_{a} n_{a}= & \frac{1}{4}\left(\hat{K}_{a b} \hat{K}_{a b}+\hat{N}_{a b} \hat{N}_{a b}\right)+\frac{3}{2} n_{a} n_{a} \\
& -\frac{1}{6}\left(K^{2}+N^{2}\right)+4 \pi G \rho,
\end{aligned}
$$

$$
D_{b} \hat{K}_{a b}=\frac{2}{3} D_{a} K-\varepsilon_{a b c} \hat{K}_{b d} \hat{N}_{d c}+3 \hat{K}_{a b} n_{b}-8 \pi G j_{a} .
$$

The $N_{a b}$ constraint Eq. (13) becomes 


$$
D_{b} \hat{N}_{a b}=\frac{2}{3} D_{a} N-\varepsilon_{a b c} D_{b} n_{c}-\frac{4}{3} N n_{a}+2 \hat{N}_{a b} n_{b} .
$$

Finally, the spatial triad variables $B_{a}{ }^{k}$ are evolved via

$$
D_{0} B_{a}{ }^{k}=-\varepsilon_{a b c} \omega_{b} B_{c}{ }^{k}-\hat{K}_{a c} B_{c}{ }^{k}-\frac{K}{3} B_{a}{ }^{k},
$$

and are constrained by

$$
\varepsilon_{a b c} D_{b} B_{c}{ }^{k}=\hat{N}_{a b} B_{b}{ }^{k}+\varepsilon_{a b c} n_{b} B_{c}{ }^{k}-\frac{2 N}{3} B_{a}{ }^{k} .
$$

\section{GAUGE CONDITIONS}

In the WEBB tetrad formalism, in addition to specifying the usual lapse function and shift vector in order to control the evolution of the coordinates, gauge conditions are required to specify the evolution of the tetrad vectors. In our hypersurface-orthogonal implementation, the acceleration vector is given in terms of the lapse by Eq. (8). The remaining freedom is the evolution of the angular velocity (with respect to Fermi-Walker frame dragging) 3-vector $\omega_{a}$. In this section, we derive the equation for the lapse function appropriate to our CMC hypersurface condition and the elliptic equations which preserve the 3D Nester gauge conditions on the spatial triad during the evolution. We assume for the present that the shift vector is given; discussion and derivation of the shift equations is deferred to Sec. V B.

\section{A. CMC slicing}

In order to fix the lapse and the corresponding foliation of space-time, we require that the trace of the extrinsic curvature be a positive constant in both space and time,

$$
K=\text { const. }>0,
$$

which with our sign convention means that the spatial hypersurfaces go to future, rather than past, null infinity. Then Eq. (18) becomes the following linear, elliptic equation for the lapse:

$$
\begin{aligned}
& {\left[-\left(D_{a}-2 n_{a}\right) D_{a}+\hat{K}_{a b} \hat{K}_{a b}\right.} \\
& \left.+\frac{1}{3} K^{2}+4 \pi G\left(\rho+\sigma_{a a}\right)\right] \alpha=0 .
\end{aligned}
$$

We notice that the operator $\left(D_{a}-2 n_{a}\right) D_{a}=\nabla_{a} \nabla_{a}$ is equal to the covariant Laplacian on the three-geometry, where here and in the following, $\nabla_{a}$ denotes the covariant derivative with respect to the three-geometry. It follows that the operator inside the square parenthesis is (formally) positive if the strong energy condition, $\rho+\sigma_{a a} \geq 0$, holds.

\section{B. 3D Nester gauge}

After imposing hypersurface orthogonality, the remaining freedom in the choice of the tetrad is described by local rotations of the spacelike legs. Nester [25] proposed a set of natural gauge conditions in order to fix this freedom up to a global rotation. These conditions are conveniently formulated using differential forms. Let $\theta^{a}, a=1,2,3$, be the coframe associated with $\mathbf{B}_{a}$; that is, $\theta^{1}, \theta^{2}, \theta^{3}$ are the basis of one-forms defined by

$$
\theta^{a}\left(\mathbf{B}_{b}\right)=\delta^{a}{ }_{b} .
$$

The Cartan structure equations (see for instance [40]) imply the following relation between $\theta^{a}$ and the connection coefficients $\Gamma_{a c d}$ :

$$
d \theta^{a}=\Gamma_{c d}^{a} \theta^{c} \wedge \theta^{d} .
$$

The 3D Nester gauge condition is ${ }^{2}$

$$
d \Xi+\delta \Pi=0,
$$

where the one-form $\Xi$ and the three-form $\Pi$ are defined by

$$
\begin{aligned}
& \Xi \equiv i_{\mathbf{B}_{a}} d \theta^{a}=\Gamma_{a b}^{a}{ }_{a b}, \\
& \Pi \equiv \frac{1}{2} \delta_{a b} \theta^{a} \wedge d \theta^{b}=\frac{1}{2} \Gamma_{b c d} \theta^{b} \wedge \theta^{c} \wedge \theta^{d},
\end{aligned}
$$

respectively. Here, $\delta$ denotes the codifferential which is defined as the formal adjoint of the exterior derivative operator $d$ with respect to the inner product $(\nu, \eta) \equiv$ $\int_{\Sigma_{t}} \nu \wedge * \eta$ for $p$-forms $\nu$ and $\eta$. In terms of the Hodge dual $*$, it is explicitly given by $\delta=(-1)^{p} * d *$ when acting on a $p$-form. Provided suitable boundary conditions are specified at the boundary of $\Sigma_{t}$, the two terms arising in Eq. (28) are mutually orthogonal with respect to the product $(\cdot, \cdot)$, and the $3 \mathrm{D}$ Nester gauge condition is equivalent to

$$
d \Xi=0, \quad d * \Pi=0 .
$$

In our notation, we have $\Xi=2 n_{a} \theta^{a}$ and $* \Pi=N$. Therefore, assuming that $\Sigma_{t}$ is connected and that its first cohomology vanishes, the 3D Nester gauge implies

$$
n_{a}=D_{a} \Phi, \quad N=\text { const. }
$$

for a function $\Phi$ on $\Sigma_{t}$. We choose the constant value of $N=0$, since this tends to minimize unnecessary twisting of the triad vectors from one spatial point to another, which is particularly appropriate in the context of asymptotic flatness. The existence and uniqueness of a set of orthonormal three-frames globally satisfying the 3D Nester gauge can be related to the existence and uniqueness of a nowhere vanishing spinor field satisfying the 3D Dirac equation, see Ref. [42] and Appendix C.

\footnotetext{
${ }^{2}$ This condition has a natural generalization to higher dimensions, see [41].
} 


\section{Properties of the evolution system in the CMC and 3D Nester gauge}

The gauge conditions we have adopted have the following nice properties: they fix the gauge variables $K, N$ and $n_{a}$ (the last up to a gradient). As a consequence, the evolution system reduces to the advection Eq. (24) for the triad vectors $\mathbf{B}_{a}$ and the Maxwell-like Eqs. (16) and (17) for the symmetric, traceless parts of $K_{a b}, N_{a b}$. This system constitutes a quasilinear, symmetric hyperbolic evolution system, assuming that the gauge variables $a_{b}=$ $D_{b}(\log \alpha), n_{b}=D_{b} \Phi$, and $\omega_{b}$ are known. The gauge variables are determined by a set of coupled elliptic equations obtained by requiring that the gauge conditions be preserved during the evolution. The lapse is obtained by solving the elliptic Eq. (27), which is a consequence of setting the time derivative of $K$ to zero, preserving CMC slicing. Next, we note that in the 3D Nester gauge, the Hamiltonian constraint (21) yields an elliptic equation for the scalar potential $\Phi$ which might be used to obtain $n_{b}$. Finally, the requirement that the time evolution preserve the 3D Nester gauge condition yields an elliptic system of equations for $2 \alpha D_{0} \Phi$ and $\alpha \omega_{b}$. This system can be derived as follows. Using the commutation relation $\left[\partial_{t}-\mathcal{L}_{\beta}, D_{a}\right] \Phi=-\alpha\left(K_{a c}+\varepsilon_{a b c} \omega_{b}\right) D_{c} \Phi$ in Eq. (20), with $n_{a}=D_{a} \Phi$, we obtain

$$
\begin{aligned}
D_{a}\left(2 \alpha D_{0} \Phi\right)+\varepsilon_{a b c} D_{b}\left(\alpha \omega_{c}\right) \\
=-\alpha\left[\varepsilon_{a b c} \hat{K}_{b d} \hat{N}_{d c}-\hat{K}_{a b}\left(3 n_{b}+a_{b}\right)\right. \\
\left.\quad+\frac{2}{3} K a_{a}+8 \pi G j_{a}\right] .
\end{aligned}
$$

In addition, with $a_{a}=D_{a}(\log \alpha)$ and $N=0$, Eq. (19) can be rewritten as

$$
D_{a}\left(\alpha \omega_{a}\right)=-\alpha \hat{N}_{a b} \hat{K}_{a b} .
$$

As shown in Appendix C, Eqs. (31) and (32) are equivalent to the inhomogeneous 3D Dirac equation, and they can be cast as a second-order elliptic system for appropriate potential fields. This elliptic formulation will be presented explicitly in the next section, after the conformal decomposition of the fields has been performed.

Summarizing, in the CMC and 3D Nester gauge conditions, our system reduces naturally to a symmetric hyperbolic evolution problem for the fields $\mathbf{B}_{a}, \hat{K}_{a b}, \hat{N}_{a b}$ which is coupled to an elliptic system which determines the gauge variables $\alpha, \Phi$ and $\omega_{a}$. Appropriate conditions on the evolution of the spatial coordinates, and the corresponding equations for the shift vector, will be discussed in Sec. V B. The well-posedness of the full, coupled hyperbolic-elliptic system depends on the structure of the elliptic equations for the gauge variables, and the choice of the shift. A well-posed Cauchy formulation has been obtained by Andersson and Moncrief [30] for a somewhat similar metric-based system.

\section{THE CONFORMALLY RESCALED EQUATIONS}

With compactified spatial coordinates and asymptotically null spatial hypersurfaces, so that $\mathcal{J}^{+}$is at a finite coordinate radius $R_{+}$, the physical space-time (coordinate) metric $g_{\mu \nu}$ is singular at $\mathcal{J}^{+}$. The spatial metric must generate infinite spatial distances over a finite range of coordinates, and the lapse becomes infinite as the hypersurface becomes null. In the conformal approach to asymptotically flat spacetimes, pioneered by Penrose [43], these infinities are absorbed into a conformal factor $\Omega$ such that the conformal metric $\tilde{g}_{\mu \nu}$, defined by

$$
g_{\mu \nu}=\Omega^{-2} \tilde{g}_{\mu \nu}
$$

is finite at $\mathcal{J}^{+}$, with $\Omega=0$ at $\mathcal{J}^{+}$and positive everywhere in the interior. The physical manifold $M$ can be considered as part of a larger conformal manifold $\tilde{M}$ containing the boundary of the physical manifold $\partial M$ as a regular null hypersurface and in which the CMC hypersurfaces are completely spacelike. The conformal metric is taken to be regular at $\mathcal{J}^{+}$, with the precise amount of smoothness there open to some debate (see Sec. I).

In the $3+1$ tetrad formalism, the above scaling of the coordinate metric under a conformal transformation corresponds to the lapse and the coordinate components of the spatial triad vectors scaling as

$$
\alpha=\Omega^{-1} \tilde{\alpha}, \quad B_{a}{ }^{i}=\Omega \tilde{B}_{a}{ }^{i} .
$$

The coordinate components of the shift vector are unchanged by the conformal transformation. We can then write for the directional derivatives

$$
D_{0}=\Omega \tilde{D}_{0}, \quad D_{a}=\Omega \tilde{D}_{a} .
$$

Note, however, that the triad components of the shift vector transform as $\tilde{\beta}^{a}=\Omega \beta^{a}$.

From the definition of the tetrad connection coefficients, Eq. (4),

$$
\Gamma_{\alpha \beta \gamma}=\Omega \tilde{\Gamma}_{\alpha \beta \gamma}+\tilde{D}_{\alpha} \Omega \eta_{\beta \gamma}-\tilde{D}_{\beta} \Omega \eta_{\alpha \gamma} .
$$

This translates into the following conformal transformations of the dyadic decompositions of the connection coefficients:

$$
N_{a b}=\Omega \tilde{N}_{a b}+\varepsilon_{a b c} \tilde{D}_{c} \Omega, \quad K_{a b}=\Omega \tilde{K}_{a b}-\tilde{D}_{0} \Omega \delta_{a b},
$$

from which

$$
\begin{gathered}
\hat{N}_{a b}=\Omega \hat{\tilde{N}}_{a b}, \quad N=\Omega \tilde{N}, \quad n_{b}=\Omega \tilde{n}_{b}+\tilde{D}_{b} \Omega, \\
\hat{K}_{a b}=\Omega \hat{\tilde{K}}_{a b}, \quad K=\Omega \tilde{K}-3 \tilde{D}_{0} \Omega,
\end{gathered}
$$

and

$a_{b}=\Omega \tilde{a}_{b}-\tilde{D}_{b} \Omega, \quad \tilde{a}_{b}=\tilde{D}_{b}(\log \tilde{\alpha}), \quad \omega_{b}=\Omega \tilde{\omega}_{b}$.

The choice of conformal scaling for the energymomentum tensor is somewhat arbitrary. The physical 
tetrad components of a radiation energy-momentum tensor are expected to scale as $\Omega^{4}$, two powers from the inverse $r^{2}$ scaling of divergent radiation in a Minkowski frame, and two redshift-time dilation factors due to our tetrad frame becoming asymptotically null. Therefore, we will define the conformal energy-momentum tensor by

$$
\tau_{\alpha \beta}=\Omega^{4} \tilde{\tau}_{\alpha \beta}, \quad \tilde{\rho} \equiv \tilde{\tau}_{00}, \quad \tilde{j}_{b} \equiv-\tilde{\tau}_{0 b}, \quad \tilde{\sigma}_{a b} \equiv \tilde{\tau}_{a b} .
$$

In principle, there is quite a bit of freedom in the choice of the conformal factor $\Omega$. Any function which is positive over the physical manifold and vanishes in a suitable way at $\mathcal{J}^{+}$would do, since a change in $\Omega$ can be compensated for by a change in the determinant of the conformal metric. One possible choice would be to demand that $\Omega^{-6}$ equal the determinant of the physical spatial metric, so the determinant of the conformal spatial metric is 1 . However, such a choice would be highly coordinate dependent. What seems to us to be a much better choice, given our use of the 3D Nester gauge, is $\Omega=e^{\Phi}$, where $\Phi$ is the Nester potential for the physical space, see Eq. (30). This has the great simplifying consequence that $\tilde{n}_{a} \equiv 0$ [26], and together with $N=0$ this means that the conformal connection coefficients $\tilde{N}_{a b}=\hat{\tilde{N}}_{a b}$ are symmetric and traceless. Another consequence is that the triad vector fields, as vector fields in the conformal 3-space, are divergenceless:

$$
\tilde{\nabla}_{k} \tilde{B}_{a}{ }^{k}=-2 \tilde{n}_{a}=0 .
$$

The covariant conformal derivative of an arbitrary vector field $\mathbf{v}=v_{a} \mathbf{B}_{a}$ is

$$
\tilde{\nabla}_{a} v_{b}=\tilde{D}_{a} v_{b}-\hat{\tilde{N}}_{a c} v_{d} \varepsilon_{c d b},
$$

and $\tilde{\nabla}_{a} v_{a}=\tilde{D}_{a} v_{a}$. Therefore, in the conformal Laplacian of a scalar or the conformal divergence of a vector field, the covariant derivatives can be replaced by directional derivatives. Furthermore, the conformal Ricci tensor is

$$
\tilde{R}_{a b}=-\tilde{D}_{c} \hat{\tilde{N}}_{d(a} \varepsilon_{b) c d}+2 \hat{\tilde{N}}_{a c} \hat{\tilde{N}}_{b c}-\delta_{a b} \hat{\tilde{N}}_{c d} \hat{\tilde{N}}_{c d} .
$$

The Ricci scalar has the simple form $\tilde{R}=-\hat{\tilde{N}}_{a b} \hat{\tilde{N}}_{a b}$ and is negative definite.

The Hamiltonian constraint Eq. (21) then becomes a relatively simple elliptic equation for $\Omega$ :

$$
\begin{aligned}
\Omega \tilde{D}_{c} \tilde{D}_{c} \Omega= & \frac{3}{2}\left[\left(\tilde{D}_{c} \Omega\right)\left(\tilde{D}_{c} \Omega\right)-\left(\frac{K}{3}\right)^{2}\right] \\
& +\frac{\Omega^{2}}{4}\left(\hat{\tilde{K}}_{c d} \hat{\tilde{K}}_{c d}+\hat{\tilde{N}}_{c d} \hat{\tilde{N}}_{c d}\right)+4 \pi G \Omega^{4} \tilde{\rho} .
\end{aligned}
$$

With the Nester-gauge inspired choice of $\Omega$, and with the time derivatives of $\Omega$ expressed in terms of $\tilde{K}$, the evolution equations for the conformal quantities are

$$
\tilde{D}_{0} \Omega=-\frac{1}{3}(K-\Omega \tilde{K}),
$$

$$
\begin{aligned}
& \tilde{D}_{0} \tilde{B}_{a}{ }^{k}=-\hat{\tilde{K}}_{a b} \tilde{B}_{b}{ }^{k}-\varepsilon_{a c d} \tilde{\omega}_{c} \tilde{B}_{d}{ }^{k}-\frac{\tilde{K}}{3} \tilde{B}_{a}{ }^{k}, \\
& \tilde{D}_{0} \hat{\tilde{N}}_{a b}+\tilde{D}_{c} \hat{\tilde{K}}_{d(a} \varepsilon_{b) c d} \\
& =\left\{2 \hat{\tilde{K}}_{c(a} \hat{\tilde{N}}_{b) c}-\frac{\tilde{K}}{3} \hat{\tilde{N}}_{a b}+2 \varepsilon_{c d(a} \hat{\tilde{N}}_{b) c} \tilde{\omega}_{d}\right. \\
& \left.+\frac{1}{\tilde{\alpha}}\left[\varepsilon_{c d(a} \hat{\tilde{K}}_{b) c} \tilde{D}_{d} \tilde{\alpha}-\tilde{D}_{(a}\left(\tilde{\alpha} \tilde{\omega}_{b)}\right)\right]\right\}^{T F},
\end{aligned}
$$

$$
\begin{aligned}
& \tilde{D}_{0} \hat{\tilde{K}}_{a b}-\tilde{D}_{c} \hat{\tilde{N}}_{d(a} \varepsilon_{b) c d} \\
& =\left\{-2 \hat{\tilde{N}}_{a c} \hat{\tilde{N}}_{b c}-\frac{\tilde{K}}{3} \hat{\tilde{K}}_{a b}+2 \varepsilon_{c d(a} \hat{\tilde{K}}_{b) c} \tilde{\omega}_{d}+\frac{1}{\tilde{\alpha}} \tilde{\nabla}_{a} \tilde{\nabla}_{b} \tilde{\alpha}\right. \\
& \left.\quad-\frac{2}{\Omega}\left[\tilde{\nabla}_{a} \tilde{\nabla}_{b} \Omega+\frac{K}{3} \hat{\tilde{K}}_{a b}\right]+8 \pi G \Omega^{2} \tilde{\sigma}_{a b}\right\}^{T F} .
\end{aligned}
$$

This system is subject to the constraint equations

$$
\begin{gathered}
\varepsilon_{a b c} \tilde{D}_{b} \tilde{B}_{c}{ }^{k}-\hat{\tilde{N}}_{a b} \tilde{B}_{b}{ }^{k}=0, \\
\tilde{D}_{b} \hat{\tilde{N}}_{a b}=0,
\end{gathered}
$$

$\tilde{D}_{b} \hat{\tilde{K}}_{a b}+\varepsilon_{a b c} \hat{\tilde{K}}_{b d} \hat{\tilde{N}}_{d c}-\frac{2}{\Omega}\left(\tilde{D}_{b} \Omega\right) \hat{\tilde{K}}_{a b}=-8 \pi G \Omega^{2} \tilde{j}_{a}$.

The conformal lapse $\tilde{\alpha}$ is determined by transforming the CMC hypersurface condition, Eq. (27), to conformal variables and then using the Hamiltonian constraint, Eq. (38), to eliminate the most singular terms at $\mathcal{J}^{+}$, giving

$$
\begin{aligned}
& \Omega \tilde{D}_{a} \tilde{D}_{a} \tilde{\alpha}-3\left(\tilde{D}_{a} \Omega\right) \tilde{D}_{a} \tilde{\alpha}+\left(\tilde{D}_{a} \tilde{D}_{a} \Omega\right) \tilde{\alpha} \\
& \quad-\frac{\Omega}{2}\left(\hat{\tilde{N}}_{a b} \hat{\tilde{N}}_{a b}+3 \hat{\tilde{K}}_{a b} \hat{\tilde{K}}_{a b}\right) \tilde{\alpha} \\
& =4 \pi G \Omega^{3}\left(3 \tilde{\rho}+\tilde{\sigma}_{c c}\right) \tilde{\alpha} .
\end{aligned}
$$

We see that in principle, $\Omega$ can be found in two ways: by solving the elliptic Eq. (38) of the Hamiltonian constraint or by integrating the evolution Eq. (39). The elliptic equation is degenerate at $\mathcal{J}^{+}$due to the hypersurface becoming null. The apparently singular terms constrain the asymptotic behavior of the solution, but in practice cause no numerical problems [14,17]. Likewise, we do not expect the apparently singular terms in the lapse Eq. (46) to impede the use of standard elliptic solvers. The time evolution equation for $\Omega$ could prove useful in reducing the frequency of solving its elliptic equation. However, as yet we have no equations for $\tilde{K}$ and $\tilde{\omega}_{a}$. Elliptic equations for these quantities come from requiring preservation of the Nester gauge during the evolution, as shown in the following subsection.

\section{A. Determination of $\tilde{\boldsymbol{K}}$ and $\tilde{\boldsymbol{\omega}}_{a}$}

Now we derive the elliptic equations for the gauge fields $\tilde{K}$ and $\tilde{\omega}_{a}$. These equations are based on Eqs. (32) and (31) 
from Sec. IVC which yield, in terms of the rescaled quantities,

$$
\begin{gathered}
-\tilde{D}_{a}\left(\tilde{\alpha} \tilde{\omega}_{a}\right)=\tilde{\alpha} \hat{\tilde{N}}_{a b} \hat{\tilde{K}}_{a b} \\
\tilde{D}_{a}\left(\frac{2}{3} \tilde{\alpha} \tilde{K}\right)+\varepsilon_{a b c} \tilde{D}_{b}\left(\tilde{\alpha} \tilde{\omega}_{c}\right)=\tilde{\alpha} \tilde{\mu}_{a},
\end{gathered}
$$

where

$$
\begin{aligned}
\tilde{\mu}_{a} \equiv & \hat{\tilde{K}}_{a b} \tilde{D}_{b}(\log \tilde{\alpha}+2 \log \Omega) \\
& -\varepsilon_{a b c} \hat{\tilde{K}}_{b d} \hat{\tilde{N}}_{d c}-8 \pi G \Omega^{2} \tilde{j}_{a} .
\end{aligned}
$$

While Eq. (47) depends only on the rescaled quantities, $\tilde{\mu}_{a}$ in Eq. (48) contains the apparently singular term involving the gradient of $\log \Omega$. However, $\tilde{\mu}_{a}$ is, in fact, finite at $\mathcal{J}^{+}$. Indeed, using the rescaled momentum constraint, Eq. (45), we may rewrite

$$
\tilde{\alpha} \tilde{\mu}_{a}=\tilde{D}_{b}\left(\tilde{\alpha} \hat{\tilde{K}}_{a b}\right)
$$

which does not involve the conformal factor. However, we prefer Eq. (49), since it does not contain derivatives of dynamical fields. In Sec. VI, we derive an explicit regular expression for the term $\hat{\tilde{K}}_{a b} \tilde{D}_{b} \log \Omega$ at $\mathcal{J}^{+}$. In Appendix A, we show that second radial derivatives of $\tilde{K}_{a b}$ are logarithmically singular at $\mathcal{J}^{+}$whenever outgoing radiation is present there.

In order to cast Eqs. (47) and (48) into a second-order elliptic system, we make the following ansatz which is motivated by the considerations at the end of Appendix C:

$$
\frac{2}{3} \tilde{\alpha} \tilde{K}=-\tilde{D}_{a} \zeta_{a}, \quad \tilde{\alpha} \tilde{\omega}_{a}=\tilde{D}_{a} \psi+\varepsilon_{a b c} \tilde{D}_{b} \zeta_{c},
$$

where $\psi$ and $\zeta_{a}$ are a scalar function and a vector field, respectively. Plugging this into Eqs. (47) and (48) and using the commutation relation $\left[\tilde{D}_{a}, \tilde{D}_{b}\right]=\varepsilon_{a b d} \hat{\tilde{N}}_{c d} \tilde{D}_{c}$, we obtain the elliptic system

$$
\begin{gathered}
-\tilde{D}_{a} \tilde{D}_{a} \psi-\hat{\tilde{N}}_{a b} \tilde{D}_{a} \zeta_{b}=\tilde{\alpha} \hat{\tilde{N}}_{a b} \hat{\tilde{K}}_{a b}, \\
-\tilde{D}_{b} \tilde{D}_{b} \zeta_{a}+\hat{\tilde{N}}_{a b} \tilde{D}_{b} \psi-\varepsilon_{a b c} \hat{\tilde{N}}_{c d} \tilde{D}_{d} \zeta_{b}=\tilde{\alpha} \tilde{\mu}_{a} .
\end{gathered}
$$

When supplemented with appropriate boundary conditions at $\mathcal{J}^{+}$, this system yields a unique solution for $\left(\psi, \zeta_{a}\right)$. We choose homogeneous Dirichlet boundary conditions for $\psi$ and the tangential components of $\zeta_{a}$. The normal component of $\zeta_{a}$, however, is determined by the boundary condition from Eq. (A31) which guarantees that the cross sections $\dot{\mathcal{J}}^{+}$remain metric two-spheres. This translates into a mixed boundary condition for the normal component of $\zeta_{a}$ (see Sec. VII below).

\section{B. Shift condition}

The shift governs the evolution of the spatial coordinates. Therefore, keeping the coordinate location of $\mathcal{J}^{+}$, where $\Omega=0$, at some fixed coordinate radius $R=R_{+}$ imposes a boundary condition on the shift. The equation for the evolution of the conformal factor is

$$
\tilde{\alpha} \tilde{D}_{0} \Omega=\left(\partial_{t}-\beta^{k} \partial_{k}\right) \Omega=-\tilde{\alpha}\left(\frac{K}{3}-\frac{\Omega}{3} \tilde{K}\right),
$$

with $\tilde{K}$ finite at $\mathcal{J}^{+}$. The requirement that the conformal factor remain zero at $\mathcal{J}^{+}, \partial_{t} \Omega \doteq 0$, implies that $\beta^{k} \partial_{k} \Omega=$ $\tilde{\beta}^{a} \tilde{D}_{a} \Omega \doteq K \tilde{\alpha} / 3$, which gives a constraint on the normal component of the shift. The outward normal vector field $\tilde{s}_{a}$ with respect to the conformal geometry is proportional ${ }^{3}$ to $-\tilde{D}_{a} \Omega$, and the normalization factor can be determined by evaluating the Hamiltonian constraint, Eq. (38), at $\mathcal{J}^{+}$. This gives

$$
\tilde{s}_{a} \doteq-\frac{3}{K} \tilde{D}_{a} \Omega
$$

so that the normal component of the shift at $\mathcal{J}^{+}$is $\tilde{s}_{a} \tilde{\beta}^{a} \doteq$ $-\tilde{\alpha}$. It also makes sense to require that the components of the shift tangent to $\mathcal{J}^{+}$vanish. This means the coordinates on $\mathcal{J}^{+}$are propagated along the null generators of $\mathcal{J}^{+}$. The complete shift boundary condition is then

$$
\tilde{\beta}_{a} \doteq-\tilde{\alpha} \tilde{s}_{a}, \quad \beta^{i} \doteq \frac{3 \tilde{\alpha}}{K} \tilde{h}^{i j} \partial_{j} \Omega=\frac{3 \tilde{\alpha}}{K} \tilde{B}_{a}^{i} \tilde{B}_{a}^{j} \partial_{j} \Omega .
$$

Satisfying this boundary condition is most straightforward if it is imposed as a boundary condition on a vector elliptic equation for the shift. This spatial coordinate gauge fixing may need to be done carefully, and here we mention a few possibilities and their main features.

The first possibility, the spatial harmonic gauge, is motivated by the metric-based formulation proved wellposed by Andersson and Moncrief [30]. With CMC foliations, this gauge casts the York version [44] of the standard Arnowitt-Deser-Misner (ADM) evolution equations [45] into a nonlinear system of wave equations which is coupled to elliptic equations for lapse and shift. Besides bringing the evolution equations into hyperbolic form, the spatial harmonic gauge has additional nice properties which were noted in [30] and exploited in their proof. Namely, the terms involving derivatives of the three-metric in the elliptic equation for the lapse are eliminated. Similarly, the terms involving second derivatives of the three-metric in the vector elliptic equation for the shift cancel out. These properties were important for consistently solving the elliptic equations to a given degree of smoothness in the rigorous proof of well-posedness for the mixed hyperbolicelliptic system in Ref. [30]. While their analysis was carried out on compact CMC slices in the absence of conformal rescaling, it motivated the choice of the spatial harmonic gauge for the conformal spatial geometry in the

\footnotetext{
${ }^{3}$ The minus sign in the expression $-\tilde{D}_{a} \Omega$ comes from the fact that $\Omega$ is strictly positive in the interior of $\Sigma_{t}$, hence, its gradient points inward from $\mathcal{J}^{+}$.
} 
conformally compactified scheme of Moncrief and Rinne [13].

In terms of the conformal metric $\tilde{h}_{i j}$ the spatial harmonic gauge sets

$$
\tilde{V}^{k} \equiv \tilde{h}^{i j}\left[\tilde{\Gamma}^{k}{ }_{i j}-\stackrel{\circ}{\Gamma}_{i j}^{k}\right]=0,
$$

where $\tilde{\Gamma}_{i j}^{k}$ and $\stackrel{\circ}{\Gamma}_{i j}^{k}$ are, respectively, the Christoffel symbols of the conformal three-metric $\tilde{h}_{i j}$ and of a given, possibly time-dependent, reference metric $\stackrel{\circ}{h}_{i j}$ on $\Sigma_{t}$. Requiring this gauge condition to remain satisfied during the evolution implies an elliptic equation for the shift, derived from $\partial_{t} \tilde{V}^{k}=0$.

In general, there is no simple way to express the Christoffel symbols in terms of the triad connection coefficients. However, in the conformal Nester gauge, there is a very simple expression for the trace $\tilde{\Gamma}^{k} \equiv \tilde{h}^{i j} \tilde{\Gamma}^{k}{ }_{i j}$ in terms of the conformal triad vectors. A straightforward calculation gives

$$
\tilde{\Gamma}^{k}=-\partial_{i} \tilde{h}^{k i}-\tilde{h}^{k i}\left(\partial_{i} \sqrt{\tilde{h}}\right) / \sqrt{\tilde{h}}=-\tilde{D}_{a} \tilde{B}_{a}{ }^{k}-\left(\tilde{\nabla}_{i} \tilde{B}_{a}{ }^{i}\right) \tilde{B}_{a}{ }^{k} .
$$

In the conformal Nester gauge, $\tilde{\nabla}_{i} \tilde{B}_{a}{ }^{i}=0$ (see Eq. (36)), so

$$
\tilde{\Gamma}^{k}=-\tilde{D}_{a} \tilde{B}_{a}^{k} .
$$

The elliptic equation for the shift comes from substituting Eq. (40) into

$$
\begin{aligned}
\left(\partial_{t}-\beta^{\ell} \partial_{\ell}\right) \tilde{V}^{k}= & -\tilde{D}_{a}\left[\left(\partial_{t}-\beta^{\ell} \partial_{\ell}\right) \tilde{B}_{a}{ }^{k}\right]-\left(\tilde{D}_{a} \beta^{i}\right) \partial_{i} \tilde{B}_{a}{ }^{k} \\
& -\left[\left(\partial_{t}-\beta^{\ell} \partial_{\ell}\right) \tilde{B}_{a}{ }^{j}\right]\left(\partial_{j} \tilde{B}_{a}{ }^{k}+2 \tilde{B}_{a}{ }^{i}{ }^{\Gamma^{k}}{ }^{k}{ }_{i j}\right) \\
& -\tilde{B}_{a}{ }^{i} \tilde{B}_{a}{ }^{j}\left(\partial_{t}-\beta^{\ell} \partial_{\ell}\right) \stackrel{\circ}{\Gamma}^{k}{ }_{i j}=0 .
\end{aligned}
$$

After using Eqs. (48)-(50) to eliminate derivatives of $\tilde{\alpha} \tilde{\omega}_{a}$ and $\tilde{\alpha} \hat{\tilde{K}}_{a b}$, the result is

$$
\begin{aligned}
& \tilde{D}_{a} \tilde{D}_{a} \beta^{k}+2 \tilde{B}_{a}{ }^{j} \stackrel{\circ}{\Gamma}^{k}{ }_{i j} \tilde{D}_{a} \beta^{i}+\left[2 \tilde{\alpha} \tilde{\mu}_{a}-\frac{1}{3} \tilde{D}_{a}(\tilde{\alpha} \tilde{K})\right] \tilde{B}_{a}{ }^{k} \\
& \quad+2 \tilde{\alpha} \tilde{K}_{a b}\left(\tilde{D}_{a} \tilde{B}_{b}{ }^{k}+\tilde{B}_{a}{ }^{i} \tilde{B}_{b}{ }^{j} \stackrel{\circ}{\Gamma}^{k}{ }_{i j}\right) \\
& \quad-\tilde{B}_{a}{ }^{i} \tilde{B}_{a}{ }^{j}\left(\partial_{t}-\beta^{\ell} \partial_{\ell}\right) \stackrel{\circ}{ }^{k}{ }_{i j}=0 .
\end{aligned}
$$

It may be significant that the spatial harmonic gauge eliminates derivatives of the triad vectors from the triad "Laplacian" operator $\tilde{D}_{a} \tilde{D}_{a}$, which is the true scalar Laplacian operator in the conformal Nester gauge. In the spatial harmonic gauge,

$$
\begin{aligned}
\tilde{D}_{a} \tilde{D}_{a} & =\tilde{B}_{a}{ }^{i} \tilde{B}_{a}{ }^{j} \frac{\partial^{2}}{\partial x^{i} \partial x^{j}}+\left(\tilde{D}_{a} \tilde{B}_{a}{ }^{k}\right) \frac{\partial}{\partial x^{k}} \\
& =\tilde{B}_{a}{ }^{i} \tilde{B}_{a}{ }^{j}\left[\frac{\partial^{2}}{\partial x^{i} \partial x^{j}}+\stackrel{\circ}{\Gamma}^{k}{ }_{i j} \frac{\partial}{\partial x^{k}}\right] .
\end{aligned}
$$

In all of our elliptic equations except the shift equation, this completely eliminates derivatives of the conformal triad vectors. Notice also that the elliptic equation for the shift required for preserving the spatial harmonic gauge, Eq. (58), does not contain second or higher derivatives of $\tilde{B}_{a}{ }^{k}$. An appropriate choice for the reference metric of the spatial harmonic gauge may well be the trivial one $\stackrel{\circ}{h}_{i j}=$ $\delta_{i j}$, for which the Christoffel symbols vanish. This can accommodate multiple black holes, as shown in the conformally flat initial data calculations of Ref. [17].

We now consider alternative coordinate gauges, based on the motivation of choosing the shift such that the spatial metric components become time-independent as the physical and presumably the conformal space-time become stationary. The spatial harmonic gauge may well have this property, but a more direct way of achieving the goal is to choose the shift to minimize a functional quadratic in time derivatives of the conformal metric. The two options of this type presented here are contingent on establishing analytically, or by successful numerical trials, that they do not lead to instabilities. We focus on minimizing the time dependence of the conformal metric, rather than the physical metric, because our conformal factor is a coordinate scalar determined by a coordinate scalar equation.

The time dependence of our conformal metric is determined by, though not equivalent to, the evolution Eq. (40) for the conformal triad fields, which is regular at $\mathcal{J}^{+}$. Using the constraint Eq. (43), we can rewrite Eq. (40) as

$\partial_{t} \tilde{B}_{a}{ }^{i}=-\left[\tilde{D}_{a} \tilde{\beta}_{b}+\varepsilon_{a c d} \tilde{\beta}_{c} \hat{\tilde{N}}_{d b}+\tilde{\alpha}\left(\tilde{K}_{a b}+\varepsilon_{a c b} \tilde{\omega}_{c}\right)\right] \tilde{B}_{b}{ }^{i}$.

The triad components of the shift depend on the conformal rescaling, hence the $\tilde{\beta}_{a}$. Equation (59) should supersede Eq. (40) as the evolution equation for the triad vectors when the shift is calculated as the $\tilde{\beta}_{a}$ rather than the $\beta^{i}$. Contracting with the dual one-forms $\tilde{\theta}^{b}{ }_{i}$ gives

$$
\begin{aligned}
\tilde{F}_{b a} & \equiv \tilde{\theta}^{b}{ }_{i} \partial_{t} \tilde{B}_{a}{ }^{i} \\
& =-\tilde{D}_{a} \tilde{\beta}_{b}-\varepsilon_{a c d} \tilde{\beta}_{c} \hat{\tilde{N}}_{d b}-\tilde{\alpha}\left(\tilde{K}_{a b}+\varepsilon_{a c b} \tilde{\omega}_{c}\right) .
\end{aligned}
$$

One should bear in mind that the determinant of the conformal metric is not time-independent in general for the Nester-based choice of conformal factor. Therefore, the straightforward way to minimize the time dependence of the conformal metric is the "minimal conformal strain" condition minimizing $\int\left[\left(\partial_{t} \tilde{h}^{i j}\right)\left(\partial_{t} \tilde{h}^{k \ell}\right) \tilde{h}_{i k} \tilde{h}_{j \ell} w \sqrt{\tilde{h}}\right] d^{3} x$, where $w$ is a weight function. Since $\tilde{h}^{i j}=\tilde{B}_{a}{ }^{i} \tilde{B}_{a}{ }^{j}$, this is equivalent to minimizing the functional

$$
I_{1}\left[\tilde{\beta}_{a}\right]=\int \tilde{F}_{(a b)} \tilde{F}_{(a b)} w \tilde{\eta},
$$

where $\tilde{\eta}=\sqrt{\tilde{h}} d^{3} x$ is the natural volume element of the conformal geometry. The trivial choice of weight function, $w=1$, seems as good as any. The symmetrization of $\tilde{F}_{a b}$ in Eq. (60) is what makes it essentially equivalent to the conventional minimal strain condition in terms of $\tilde{h}^{i j}$. 
With

$$
\tilde{F}_{(a b)}=-\tilde{\nabla}_{(a} \tilde{\beta}_{b)}-\tilde{\alpha} \tilde{K}_{a b},
$$

where $\tilde{\nabla}_{a}$ is the conformal covariant derivative operator, minimizing $I_{1}$ gives the elliptic equation

$$
-\tilde{\nabla}_{b} \tilde{F}_{(a b)}=\frac{1}{2}\left(\tilde{\nabla}_{b} \tilde{\nabla}_{b} \tilde{\beta}_{a}+\tilde{\nabla}_{b} \tilde{\nabla}_{a} \tilde{\beta}_{b}\right)+\tilde{\nabla}_{b}\left(\tilde{\alpha} \tilde{K}_{a b}\right)=0,
$$

and the boundary variation $\delta \tilde{\beta}_{a} \tilde{F}_{(a b)} \tilde{s}_{b}$ vanishing is consistent with the Dirichlet boundary condition (55) at $\mathcal{J}^{+}$ discussed above. As in the previous subsection, we prefer avoiding radial derivatives of the fields in the source terms of the elliptic equations. For this reason, we use the momentum constraint Eq. (45), and substitute

$$
\begin{aligned}
\tilde{\nabla}_{b}\left(\tilde{\alpha} \tilde{K}_{a b}\right)= & \tilde{\alpha}\left[\hat{\tilde{K}}_{a b} \tilde{D}_{b}(\log \tilde{\alpha}+2 \log \Omega)-8 \pi G \Omega^{2} \tilde{j}_{a}\right] \\
& +\frac{1}{3} \tilde{D}_{a}(\tilde{\alpha} \tilde{K}) .
\end{aligned}
$$

As mentioned before, a regular expression for the term $\hat{\tilde{K}}_{a b} \tilde{D}_{b} \log \Omega$ at $\mathcal{J}^{+}$will be derived in Sec. VI. We note that expanding the covariant derivatives gives

$$
\begin{aligned}
\tilde{\nabla}_{b} \tilde{\nabla}_{b} \tilde{\beta}_{a}= & \tilde{D}_{b} \tilde{D}_{b} \tilde{\beta}_{a}+2 \varepsilon_{a c d} \hat{\tilde{N}}_{b d} \tilde{D}_{b} \tilde{\beta}_{c}+\hat{\tilde{N}}_{a b} \hat{\tilde{N}}_{b c} \tilde{\beta}_{c} \\
& -\hat{\tilde{N}}_{b c} \hat{\tilde{N}}_{b c} \tilde{\beta}_{a}
\end{aligned}
$$

and

$$
\tilde{\nabla}_{b} \tilde{\nabla}_{a} \tilde{\beta}_{b}=\tilde{\nabla}_{a}\left(\tilde{\nabla}_{b} \tilde{\beta}_{b}\right)+\tilde{R}_{a b} \tilde{\beta}_{b}=\tilde{D}_{a}\left(\tilde{D}_{b} \tilde{\beta}_{b}\right)+\tilde{R}_{a b} \tilde{\beta}_{b},
$$

where the constraint Eq. (44) has been used and $\tilde{R}_{a b}$ is given by Eq. (37).

Smarr and York [46] argue that a different shift condition, the "minimal distortion" condition, most cleanly suppresses that part of the time dependence of the spatial metric associated with diffeomorphisms of the spatial coordinates. This minimizes the time dependence of what they call the conformal spatial metric, $\hat{h}^{i j} \equiv\left(h^{1 / 3}\right) h^{i j}$, by minimizing the action $\int\left[\left(\partial_{t} \hat{h}^{i j}\right)\left(\partial_{t} \hat{h}^{k \ell}\right) \hat{h}_{i k} \hat{h}_{j \ell} \sqrt{h}\right] d^{3} x$. Their rationale for minimal distortion rather than minimal strain is based on using the Hamiltonian constraint to determine the determinant of the spatial metric, but we make a quite different kind of conformal decomposition. For completeness, we note that the minimal distortion shift can be implemented in our formalism by defining $\hat{B}_{a}{ }^{i} \equiv$ $B_{a}{ }^{i} /(\operatorname{det} B)=\tilde{B}_{a}{ }^{i} /(\operatorname{det} \tilde{B})$ and $\hat{\widetilde{F}}_{a b} \equiv \tilde{F}_{a b}-\frac{1}{3} \delta_{a b} \tilde{F}_{c c}$. The minimal distortion version of the action is

$$
I_{2}\left[\tilde{\beta}_{a}\right]=\int \hat{\tilde{F}}_{(a b)} \hat{\tilde{F}}_{(a b)} \tilde{\eta} .
$$

The differential equation for the shift is then

$$
\begin{aligned}
0 & =-\tilde{\nabla}_{b} \tilde{\tilde{F}}_{(a b)} \\
& =\frac{1}{2}\left(\tilde{\nabla}_{b} \tilde{\nabla}_{b} \tilde{\beta}_{a}+\tilde{\nabla}_{b} \tilde{\nabla}_{a} \tilde{\beta}_{b}\right)-\frac{1}{3} \tilde{\nabla}_{a} \tilde{\nabla}_{b} \tilde{\beta}_{b}+\tilde{\nabla}_{b}\left(\tilde{\alpha} \hat{\tilde{K}}_{a b}\right) \\
& =\frac{1}{2}\left(\tilde{\nabla}_{b} \tilde{\nabla}_{b} \tilde{\beta}_{a}+\frac{1}{3} \tilde{\nabla}_{a} \tilde{\nabla}_{b} \tilde{\beta}_{b}+\tilde{R}_{a b} \tilde{\beta}_{b}\right)+\tilde{\nabla}_{b}\left(\tilde{\alpha} \tilde{\tilde{K}}_{a b}\right),
\end{aligned}
$$

and the boundary variation is $\delta \tilde{\beta}_{a} \hat{\tilde{F}}_{(a b)} \tilde{s}_{b}$, which vanishes for the Dirichlet boundary condition (55).

All of the above forms of the shift equation are regular at $\mathcal{J}^{+}$and should give rise to a well-posed elliptic boundary value problem on a CMC hypersurface (with other variables fixed) as long as the only boundary is at $\dot{\mathcal{J}}^{+}$. If black holes are present, there should also be inner boundaries at excision surfaces inside or at the apparent horizons, but we defer discussion of inner boundary conditions to a future paper.

\section{REGULARITY CONDITIONS AT $\mathcal{J}^{+}$}

The evolution Eqs. (39)-(41) for $\Omega, \tilde{B}_{a}{ }^{k}$ and $\hat{\tilde{N}}_{a b}$ are completely regular at $\mathcal{J}^{+}$. However, Eq. (42) for the evolution of $\hat{\tilde{K}}_{a b}$ has the apparently singular term

$$
S_{a b} \equiv \frac{1}{\Omega}\left(\tilde{\nabla}_{a} \tilde{\nabla}_{b} \Omega+\frac{K}{3} \hat{\tilde{K}}_{a b}\right)^{T F}
$$

at $\mathcal{J}^{+}$. In this section, we derive the appropriate regularity conditions which ensure that $S_{a b}$ is finite at $\mathcal{J}^{+}$. To analyze the limit of the covariant Hessian $\tilde{\Xi}_{a b} \equiv \tilde{\nabla}_{a} \tilde{\nabla}_{b} \Omega=$ $\tilde{\nabla}_{(a} \tilde{\nabla}_{b)} \Omega$ at $\mathcal{J}^{+}$, we introduce the tensor $\tilde{\gamma}_{a b} \equiv$ $\delta_{a b}-\tilde{s}_{a} \tilde{s}_{b}$ projecting tangent to $\dot{\mathcal{J}}^{+}$and the $2 \mathrm{D}$ extrinsic curvature of $\dot{J}^{+}$

$$
\tilde{\kappa}_{a b} \equiv \tilde{\gamma}_{a c} \tilde{\gamma}_{b d} \tilde{\nabla}_{c} \tilde{s}_{d}=\tilde{\gamma}_{a c} \tilde{\nabla}_{c} \tilde{s}_{b},
$$

with a similar sign convention to that of the $3 \mathrm{D}$ extrinsic curvature, such that $\tilde{\kappa} \equiv \tilde{\kappa}_{c c}$ is positive for expansion of the outward normals. With $\tilde{s}_{b} \doteq-(3 / K) \tilde{\nabla}_{b} \Omega$ and smoothly extending $\tilde{s}_{b}$ as the tangent vector to the normal spacelike geodesic in a neighborhood of $\dot{J}^{+}$, decomposing $\tilde{\Xi}_{a b}$ into components along and perpendicular to $\tilde{s}_{a}$ gives

$$
\begin{aligned}
\tilde{\Xi}_{a b} \doteq & \tilde{s}_{a} \tilde{s}_{b} \tilde{s}_{c} \tilde{s}_{d} \tilde{\nabla}_{c} \tilde{\nabla}_{d} \Omega+\tilde{s}_{a} \tilde{s}_{c} \tilde{\gamma}_{b d} \tilde{\nabla}_{d} \tilde{\nabla}_{c} \Omega \\
& +\tilde{s}_{b} \tilde{s}_{d} \tilde{\gamma}_{a c} \tilde{\nabla}_{c} \tilde{\nabla}_{d} \Omega+\tilde{\gamma}_{a c} \tilde{\gamma}_{b d} \tilde{\nabla}_{c} \tilde{\nabla}_{d} \Omega \\
\doteq & \tilde{s}_{a} \tilde{s}_{b} \tilde{s}_{c} \tilde{\nabla}_{c}\left(\tilde{s}_{d} \tilde{\nabla}_{d} \Omega\right)-\frac{K}{3} \tilde{\kappa}_{a b},
\end{aligned}
$$

since $\tilde{s}_{c} \tilde{\nabla}_{c} \tilde{s}_{d}=0, \tilde{\kappa}_{b c} \tilde{s}_{c}=0$ and $\Omega \doteq 0$. Take the trace of Eq. (65) to get

$$
\tilde{\nabla}_{c} \tilde{\nabla}_{c} \Omega \doteq \tilde{s}_{c} \tilde{\nabla}_{c}\left(\tilde{s}_{d} \tilde{\nabla}_{d} \Omega\right)-\frac{K}{3} \tilde{\kappa},
$$

and the gradient of Eq. (38) gives 


$$
\tilde{s}_{b} \tilde{\Xi}_{a b} \doteq \frac{1}{3} \tilde{s}_{a} \tilde{D}_{b} \tilde{D}_{b} \Omega \doteq \frac{1}{3} \tilde{s}_{a} \tilde{s}_{c} \tilde{\nabla}_{c}\left(\tilde{s}_{d} \tilde{\nabla}_{d} \Omega\right)-\frac{K}{9} \tilde{\kappa} \tilde{s}_{a},
$$

from which

$$
\tilde{s}_{c} \tilde{\nabla}_{c}\left(\tilde{s}_{d} \tilde{\nabla}_{d} \Omega\right)=\tilde{s}_{c} \tilde{s}_{d} \tilde{\Xi}_{a b} \doteq-\tilde{\kappa} K / 6
$$

and

$$
\tilde{\Xi}_{a b} \doteq-\frac{K}{3}\left(\tilde{\kappa}_{a b}+\frac{1}{2} \tilde{\kappa} \tilde{s}_{a} \tilde{s}_{b}\right) .
$$

Therefore, we find for the singular term in Eq. (42)

$$
\begin{aligned}
\Omega S_{a b} & =\left(\tilde{\Xi}_{a b}\right)^{T F}+\frac{K}{3} \hat{\tilde{K}}_{a b} \\
& \doteq-\frac{K}{3}\left(\tilde{\kappa}_{a b}-\frac{1}{2} \tilde{\gamma}_{a b} \tilde{\kappa}-\hat{\tilde{K}}_{a b}\right) .
\end{aligned}
$$

To ensure that $S_{a b}$ is finite, we see that the condition

$$
\hat{\tilde{\kappa}}_{a b} \equiv \tilde{\kappa}_{a b}-\frac{1}{2} \tilde{\gamma}_{a b} \tilde{\kappa} \doteq \hat{\tilde{K}}_{a b}
$$

must hold at $\dot{\mathcal{J}}^{+}$, and both

$$
\hat{\tilde{K}}_{a b} \tilde{s}_{b}=O(\Omega)
$$

and

$$
\hat{\tilde{\kappa}}_{a b}-\tilde{\gamma}_{a c} \tilde{\gamma}_{b d} \hat{\tilde{K}}_{c d}=O(\Omega)
$$

at $\dot{\mathcal{J}}^{+}$.

The first of these conditions, that only the transversetraceless part of $\tilde{K}_{a b}$ can be nonzero at $\dot{\mathcal{J}}^{+}$, is what is required to resolve the apparently singular terms in the sources for the elliptic equations discussed in Sec. V. As we show at the end of this section, the limit of $\Omega^{-1} \hat{\tilde{K}}_{a b} \tilde{s}_{b}$ at $\mathcal{J}^{+}$can be derived from the momentum constraint equation [27]. The second condition, Eq. (69), is known as the zero-shear condition, and physically is a constraint on the initial data that limits the amplitude of incoming waves in the neighborhood of $\mathcal{J}^{+}$and ultimately at past null infinity. There is an extensive literature (see [27]) showing how these conditions relate to the existence of $\mathcal{J}^{+}$as a regular null hypersurface in the conformal space-time.

While Eq. (66) can be inverted to get an expression for $\tilde{\kappa}_{a b}$ at $\dot{\mathcal{J}}^{+}$in terms of directional derivatives of $\Omega$, it is better to evaluate $\tilde{\kappa}_{a b}$ from its defining expression

$$
\tilde{\kappa}_{a b}=\tilde{\gamma}_{a c} \tilde{\nabla}_{c} \tilde{s}_{b}=\tilde{\gamma}_{a c}\left(\tilde{D}_{c} \tilde{s}_{b}+\varepsilon_{b d e} \tilde{s}_{d} \hat{\tilde{N}}_{c e}\right),
$$

which does not contain any second normal derivatives of $\Omega$. Since the directional derivative of $\tilde{s}_{b}$ is tangent to $\dot{J}^{+}$, $\tilde{\kappa}_{a b}$ at $\dot{\mathcal{J}}^{+}$is obtained by substituting $\tilde{s}_{b}=-(3 / K) \tilde{D}_{b} \Omega$ into Eq. (70). The trace of the $2 \mathrm{D}$ extrinsic curvature is then

$$
\tilde{\kappa}=\tilde{\gamma}_{a b} \tilde{D}_{b} \tilde{s}_{a} \doteq-\frac{3}{K} \tilde{\gamma}_{a b} \tilde{D}_{b} \tilde{D}_{a} \Omega .
$$

Away from $\dot{\mathcal{J}}^{+}, \tilde{s}_{b}$ is found by integrating the geodesic equation.

In the following, we compute an explicitly finite expression at $\mathcal{J}^{+}$for the apparently singular term $S_{a b}$ when the regularity conditions (68) and (69) are satisfied. The direct approach using l'Hôpital's rule would require going to higher order in the asymptotic expansions, which is awkward in the context of the tetrad equations. The Nester gauge conditions, as elliptic equations which are regular at $\mathcal{J}^{+}$, impose no local constraints on the behavior of the $\hat{\tilde{N}}_{a b}$ near or at $\mathcal{J}^{+}$. On the other hand, the asymptotic expansions are rather straightforward when dealing with coordinate components in certain special coordinate systems. We derive in Appendix A the asymptotic expansion of the conformal factor in Gaussian normal coordinates, and from this, expressions for the coordinate components $S_{i j}$. Since $S$ is a 3-tensor, it is then trivial to obtain the corresponding expression for the triad components, though evaluating this expression is not so easy, since it requires knowing $\tilde{s}_{a}$ away from $\dot{J}^{+}$.

An alternative approach is to impose the Penrose regularity condition that the Weyl tensor vanish at $\mathcal{J}^{+}$. In Appendix A, we use the asymptotic expansions in Gaussian normal coordinates to evaluate the Weyl tensor and determine the additional constraint on the asymptotic behavior of the extrinsic curvature beyond the zero-shear condition required to satisfy the Penrose condition. While it is still somewhat controversial, we believe that the Penrose condition is reasonable, in that it is only a slightly stronger restriction on the amplitude of incoming waves near $\mathcal{J}^{+}$in the initial data than the zero-shear condition, and is preserved in the subsequent evolution.

A standard expression for the electric part of the physical Weyl tensor, in which time derivatives have been eliminated using the evolution equations (as in Ref. [13], Eq. (5.1)), is

$$
\begin{aligned}
E_{a b} & =C_{0 a 0 b}=\left[R_{a b}-K_{a c} K_{c b}+K K_{a b}-4 \pi G \sigma_{a b}\right]^{T F} \\
& =\left[R_{a b}-\hat{K}_{a c} \hat{K}_{c b}+\frac{K}{3} \hat{K}_{a b}-4 \pi G \sigma_{a b}\right]^{T F}
\end{aligned}
$$

The conformal invariance of the Weyl tensor means that the conformal Weyl tensor, i.e., the Weyl tensor calculated from the full space-time conformal metric, is $\tilde{E}_{a b}=$ $\Omega^{-2} E_{a b}$. It is the coordinate components $E_{i j}$ which are truly invariant, and the scaling relation between physical and conformal triad vectors introduces the $\Omega^{-2}$ factor. In our conformal variables, and taking advantage of the simplifications of the Nester triad gauge, we have 


$$
\begin{aligned}
\tilde{E}_{a b}= & \Omega^{-2} E_{a b} \\
= & \frac{1}{\Omega}\left[\tilde{\nabla}_{a} \tilde{\nabla}_{b} \Omega+\frac{K}{3} \hat{\tilde{K}}_{a b}\right]^{T F} \\
& +\left[\tilde{R}_{a b}-\hat{\tilde{K}}_{a c} \hat{\tilde{K}}_{b c}-4 \pi G \Omega^{2} \tilde{\sigma}_{a b}\right]^{T F} \\
= & S_{a b}-\tilde{D}_{c} \hat{\tilde{N}}_{d(a} \varepsilon_{b) c d} \\
& +\left[2 \hat{\tilde{N}}_{a c} \hat{\tilde{N}}_{b c}-\hat{\tilde{K}}_{a c} \hat{\tilde{K}}_{b c}-4 \pi G \Omega^{2} \tilde{\sigma}_{a b}\right]^{T F} .
\end{aligned}
$$

Note that the expression in Eq. (72) is not conformally invariant, since the Einstein equations used to transform the electric tensor to that form are not conformally invariant. The Penrose regularity condition implies that $\widetilde{E}_{a b} \doteq 0$, such that

$$
S_{a b} \doteq \tilde{D}_{c} \hat{\tilde{N}}_{d(a} \varepsilon_{b) c d}-\left[2 \hat{\tilde{N}}_{a c} \hat{\tilde{N}}_{b c}-\hat{\tilde{K}}_{a c} \hat{\tilde{K}}_{b c}\right]^{T F} .
$$

Introduced into the evolution Eq. (42) for $\hat{\tilde{K}}_{a b}$, the result is

$$
\begin{aligned}
\tilde{D}_{0} \hat{\tilde{K}}_{a b} \doteq & -\tilde{D}_{c} \hat{\tilde{N}}_{d(a} \varepsilon_{b) c d}+\left[2\left(\hat{\tilde{N}}_{a c} \hat{\tilde{N}}_{b c}-\hat{\tilde{K}}_{a c} \hat{\tilde{K}}_{b c}\right)\right. \\
& \left.-\frac{\tilde{K}}{3} \hat{\tilde{K}}_{a b}+2 \varepsilon_{c d(a} \hat{\tilde{K}}_{b) c} \tilde{\omega}_{d}+\frac{1}{\tilde{\alpha}} \tilde{\nabla}_{a} \tilde{\nabla}_{b} \tilde{\alpha}\right]^{T F} .
\end{aligned}
$$

The conformal momentum constraint, Eq. (45), can be used to obtain more explicit information on the asymptotic behavior of the extrinsic curvature. Start from the equation in the form $\Omega \tilde{\nabla}_{b}\left(\Omega^{-1} \hat{\tilde{K}}_{a b}\right)-\Omega^{-1}\left(\tilde{\nabla}_{b} \Omega\right) \hat{\tilde{K}}_{a b}=$ $-8 \pi G \Omega^{2} \tilde{j}_{a}$. Decompose $\hat{\tilde{K}}_{a b}$ into longitudinal and transverse parts relative to the normal vector $\tilde{s}_{a}$ at $\mathcal{J}^{+}$,

$$
\hat{\tilde{K}}_{a b}=\tilde{s}_{a} \tilde{s}_{b}\left(\tilde{s}_{c} \tilde{s}_{d} \hat{\tilde{K}}_{c d}\right)+2 \tilde{s}_{(a}\left(\tilde{\gamma}_{b) d} \tilde{s}_{c} \hat{\tilde{K}}_{c d}\right)+\left(\tilde{\gamma}_{a c} \tilde{\gamma}_{b d} \hat{\tilde{K}}_{c d}\right) .
$$

Substituting into the momentum constraint and using the properties of $\tilde{s}_{a}$ gives

$$
\begin{aligned}
\tilde{s}_{a}[ & \left(\Omega \tilde{s}_{b} \tilde{\nabla}_{b}+\Omega \tilde{\kappa}-\tilde{s}_{b} \tilde{D}_{b} \Omega\right)\left(\frac{\tilde{s}_{c} \tilde{s}_{d} \hat{\tilde{K}}_{c d}}{\Omega}\right) \\
& \left.+\left(\Omega \tilde{\nabla}_{b}-\tilde{D}_{b} \Omega\right)\left(\frac{\tilde{s}_{c} \tilde{\gamma}_{b d} \hat{\tilde{K}}_{c d}}{\Omega}\right)\right] \\
& +\left(\Omega \tilde{s}_{b} \tilde{\nabla}_{b}+\Omega \tilde{\kappa}-\tilde{s}_{b} \tilde{D}_{b} \Omega\right)\left(\frac{\tilde{s}_{c} \tilde{\gamma}_{a d} \hat{\tilde{K}}_{c d}}{\Omega}\right) \\
& +\tilde{\kappa}_{a b}\left(\tilde{s}_{c} \tilde{\gamma}_{b d} \hat{\tilde{K}}_{c d}\right)+\left(\tilde{\nabla}_{b}-2 \frac{\tilde{D}_{b} \Omega}{\Omega}\right)\left(\tilde{\gamma}_{a c} \tilde{\gamma}_{b d} \hat{\tilde{K}}_{c d}\right) \\
= & -8 \pi G \Omega^{2} \tilde{j}_{a} .
\end{aligned}
$$

The purely or partially longitudinal parts of $\tilde{K}_{a b} / \Omega$ are finite at $\dot{J}^{+}$, though we shall see in Appendix A that their radial derivatives are logarithmically divergent. Despite this, those derivative terms vanish at $\dot{\mathcal{J}}^{+}$, since they contain an $\Omega$ factor in front. Projecting along $\tilde{s}_{a}$ and evaluating at $\dot{\mathcal{J}}^{+}$yields

$$
\Omega^{-1} \tilde{s}_{c} \tilde{s}_{d} \hat{\tilde{K}}_{c d} \doteq \frac{3}{K} \tilde{\kappa}_{a b}\left(\tilde{\gamma}_{a c} \tilde{\gamma}_{b d} \hat{\tilde{K}}_{c d}\right)=\frac{3}{K} \tilde{\kappa}_{c d} \hat{\tilde{K}}_{c d} .
$$

Since $\tilde{\gamma}_{c d} \hat{\tilde{K}}_{c d}=-\tilde{s}_{c} \tilde{s}_{d} \hat{\tilde{K}}_{c d}$, which is zero at $\mathcal{J}^{+}, \tilde{\kappa}_{a b}$ can be replaced by its traceless part $\hat{\tilde{\kappa}}_{a b}=\tilde{\kappa}_{a b}-\frac{1}{2} \tilde{\gamma}_{a b} \tilde{\kappa}$ in Eq. (75). The projection perpendicular to $\tilde{s}_{a}$ gives at $\mathcal{J}^{+}$

$$
\Omega^{-1} \tilde{s}_{c} \tilde{\gamma}_{a d} \hat{\tilde{K}}_{c d} \doteq-\frac{3}{K} \tilde{\gamma}_{a e} \tilde{\gamma}_{b d} \tilde{\nabla}_{b}\left(\tilde{\gamma}_{c e} \hat{\tilde{K}}_{c d}\right) .
$$

Combining these results, the $\Omega^{-1} \tilde{s}_{b} \tilde{\tilde{K}}_{b a}$ term that appears in various equations can be explicitly evaluated as

$$
\Omega^{-1} \tilde{s}_{b} \hat{\tilde{K}}_{b a} \doteq-\frac{3}{K} \tilde{\gamma}_{a e} \tilde{\gamma}_{b d} \tilde{\nabla}_{b}\left(\tilde{\gamma}_{e c} \hat{\tilde{K}}_{c d}\right)+\frac{3}{K} \tilde{s}_{a} \hat{\tilde{\kappa}}_{b c} \hat{\tilde{K}}_{b c} .
$$

Based on the asymptotic expansions in Gaussian normal coordinates, we show in Appendix A that the regularity conditions $\hat{\tilde{K}}_{a b} \tilde{s}_{b} \doteq 0$ and $\tilde{\gamma}_{a c} \tilde{\gamma}_{b d} \hat{\tilde{K}}_{c d} \doteq \hat{\tilde{\kappa}}_{a b}$ are preserved by the time evolution.

\section{SUMMARY}

The mixed hyperbolic-elliptic system proposed in this paper for solving the Einstein equations on CMC hypersurfaces in a conformally compactified asymptotically flat space-time is, we believe, worth testing as a way of improving the accuracy of numerical calculations of gravitational radiation emitted in mergers of black holes. There is no need to use artificial boundary conditions at a finite radius when the computational domain extends all the way to future null infinity, and asymptotic gravitational wave amplitudes are calculated directly, without the need for any extrapolation procedure. A purely hyperbolic system would possibly be more computationally efficient, since there are no elliptic equations to solve, but requires extra care in constructing a numerical scheme to suppress errors in the additional constraints among the additional variables such a system entails.

Our representation of tensors by tetrad, rather than coordinate, components is perhaps more controversial. We fix the timelike vector of the tetrad by requiring it to be orthogonal to the CMC hypersurfaces of constant coordinate time, but there is gauge freedom in the orientation of the triad of spatial vectors within the hypersurface, in addition to the gauge freedom in the evolution of the spatial coordinates. A key part of our scheme is combining what we consider to be the "natural" choice of triad gauge, the 3D Nester gauge, with the choice of conformal gauge (definition of the conformal factor), such that the nontrivial spatial triad connection coefficients for the conformal geometry of the CMC hypersurfaces are equivalent to the five independent components of the traceless symmetric $\hat{\tilde{N}}_{a b}$. The hyperbolic part of our evolution system for the conformally rescaled variables consists of the coupled evolution Eqs. (41) and (42) for the $\hat{\tilde{N}}_{a b}$ and the traceless part of the conformal extrinsic curvature $\hat{\tilde{K}}_{a b}$, respectively, 
plus the simple advection Eq. (40) (or (59) when $\tilde{\beta}_{a}$ is used) for the nine coordinate components of the conformal triad vectors, $\tilde{B}_{a}{ }^{i}$. This is a total of 19 evolution equations.

The remaining six tetrad connection coefficients-the triad components of the acceleration and angular velocity (relative to Fermi-Walker transport) of the tetrad framesare determined by elliptic equations: Eq. (46) for the conformal lapse, whose logarithmic gradient is the conformal acceleration, and Eqs. (51) and (52) for a scalar potential $\psi$ and a vector potential $\zeta_{a}$, which together determine the conformal angular velocity $\tilde{\omega}_{a}$ and the trace of the conformal extrinsic curvature $\tilde{K}$. The conformal gauge is enforced by the Hamiltonian constraint Eq. (38) for the conformal factor $\Omega$, but some interpolation may be possible using the evolution Eq. (39). The coordinate gauge conditions are fixed by elliptic equations for the triad components of the conformal shift. We suggest three options, Eqs. (58), (61), and (63), but some experimentation will be necessary to determine which of these, or perhaps some quite different scheme, works best in practice. We do not propose explicitly imposing the momentum constraints during the evolution, since this does not seem to be necessary for stability [14], and we similarly allow the $\tilde{D}_{b} \hat{\tilde{N}}_{a b}=0$ constraint to evolve freely. We analyze the propagation of errors in these and other constraints in Appendix B.

No boundary conditions are necessary at $\mathcal{J}^{+}$for the evolution equations, which is a major advantage of the CMC hypersurface condition. Boundary conditions are required for the elliptic equations. Fundamental to the conformal decomposition is the boundary condition $\Omega \doteq$ 0 on the conformal factor. As discussed in Sec. VB, the boundary condition at $\dot{\mathcal{J}}^{+}$on the shift vector is $\tilde{\beta}_{a} \doteq$ $-\tilde{\alpha} \tilde{s}_{a}$, where $\tilde{s}_{a}=-(3 / K) \tilde{D}_{a} \Omega$ is the unit normal to $\dot{J}^{+}$in the conformal 3-space. This ensures that $\mathcal{J}^{+}$is at a fixed coordinate radius $R_{+}$and that the angular coordinates in $\dot{J}^{+}$are propagated along the null generators of $\mathcal{J}^{+}$. These angular coordinates are related to the nominally Cartesian computational coordinates in the same way as in flat space.

The boundary conditions for the Nester gauge potentials $\psi$ and $\zeta_{a}$ introduced in Sec. VA are naturally chosen to be homogeneous Dirichlet conditions for $\psi$ and the part of $\zeta_{a}$ tangent to $\dot{\mathcal{J}}^{+}$:

$$
\psi \doteq 0, \quad \tilde{\gamma}_{a b} \zeta_{b} \doteq 0,
$$

since we do not want the conformal triad angular velocity $\tilde{\omega}_{a}$ to be unnecessarily nonzero. For a flat conformal spatial geometry, $\hat{\tilde{N}}_{a b}$ and therefore the source terms for $\psi$ are zero, which should make $\psi=0$ globally. Furthermore, in a spherically symmetric space-time, the boundary conditions should allow $\zeta_{a}$ to be spherically symmetric, which means it does not contribute to $\tilde{\omega}_{a}$.

In order to keep the intrinsic geometry of the $\dot{\mathcal{J}}^{+}$ 2 -surface that of a true sphere, with an inverse circum- ferential radius $\xi_{0}$ constant in time, as discussed in Appendix A, the boundary condition (A31) on $\tilde{K}$ must be satisfied. In terms of the Nester vector potential, this becomes $\tilde{D}_{a} \zeta_{a} \doteq-\tilde{\alpha} \tilde{\kappa}$, or

$$
\tilde{s}_{b} \tilde{D}_{b}\left(\tilde{s}_{a} \zeta_{a}\right)-\tilde{\kappa} \tilde{s}_{a} \zeta_{a} \doteq-\tilde{\alpha} \tilde{\kappa} .
$$

While we use Gaussian normal coordinates to derive this condition, it is valid regardless of the computational coordinate system since it is expressed in terms of spatial coordinate scalars. The area of the $\dot{J}^{+} 2$-sphere is then constant in time, which means that the expansion of $\mathcal{J}^{+}$ vanishes. Note that $\xi_{0}$ is not necessarily equal to the inverse of the coordinate radius of $\mathcal{J}^{+}, R_{+}$. The geometrical definition of $\xi_{0}$ is that the 2D scalar curvature of $\dot{\mathcal{J}}^{+}$ is $2 \xi_{0}{ }^{2}$. In Sec. VIII, we use the zero expansion result, plus the fact that our boundary conditions on the shift maintain a simple relation between our computational coordinates and standard polar angle coordinates on $\dot{\mathcal{J}}^{+}$, to obtain a simple expression for the Bondi news function in terms of our variables.

Finally, we consider the boundary condition on the conformal lapse. We can make our time coordinate correspond to retarded Minkowski time at $\mathcal{J}^{+}$by imposing the boundary condition

$$
\tilde{\alpha} \doteq \frac{K}{3 \xi_{0}} \equiv \tilde{\alpha}_{0}
$$

on Eq. (46) for the conformal lapse, since CMC slicing of Minkowski space has $\alpha \sim K r / 3$ as $r \rightarrow \infty$. Since $\xi_{0}$ is uniform on all of the $\mathcal{J}^{+}$hypersurface to the future of the initial hypersurface, so is $\tilde{\alpha}_{0}$.

The question of what boundary conditions to impose on an excision surface on or inside the apparent horizon of a black hole is left open for the present.

\section{DISCUSSION}

An important issue when formulating the equations on CMC hypersurfaces is dealing with singularities, real or apparent, which are associated with the hypersurfaces becoming asymptotically null as they approach $\mathcal{J}^{+}$. The elliptic equations for the conformal factor $\Omega$ and the conformal lapse $\tilde{\alpha}$ are genuinely singular there. However, the singular terms in these equations do not cause any singularity in the solutions; instead they force any solutions which satisfy the boundary conditions at $\dot{J}^{+}$to have very particular asymptotic behaviors. These asymptotic behaviors, additionally constrained by the usual "zero-shear" and Penrose regularity conditions, were discussed in Sec. VI. We also found it useful to explore the asymptotic behavior at greater depth in a special coordinate system, Gaussian normal coordinates (Appendix A). These coordinates are not suitable as global coordinates in an actual numerical calculation, but their simplicity in a neighborhood of $\dot{J}^{+}$facilitates drawing general conclusions about 
the asymptotic behavior of scalars and complete tensors. In this way, we are able to analyze the apparently singular terms that appear in some of the evolution equations, show that they are in fact finite at $\mathcal{J}^{+}$, and evaluate their limits at $\dot{\mathcal{J}}^{+}$. However, the conformal factor, the conformal lapse, and the conformal extrinsic curvature are not completely smooth at $\dot{\mathcal{J}}^{+}$on a CMC hypersurface when any outgoing radiation is present. The fourth normal derivative of $\Omega$ and the second normal derivative of the conformal extrinsic curvature are logarithmically infinite there. This is a gauge artifact of the CMC slicing condition, has nothing to do with our spatial gauge conditions, and is not prevented by any physically allowable regularity conditions. In principle, it should slow the convergence of a pseudospectral numerical scheme or a high-order finite difference scheme, but the impact may not be severe. The only apparent way of avoiding this potential problem is to make the lapse evolve dynamically as part of the hyperbolic system.

It is instructive to give a more detailed comparison of our system with the coordinate-based system of Moncrief and Rinne [13]. They have a set of 11 evolution equations for the six components of the conformal spatial metric and the five components of the traceless part of the canonical momentum $\pi^{\mathrm{tr} i j}$. The traceless canonical momentum is, within a factor of the square root of the determinant of the conformal metric, equivalent to our $\hat{\tilde{K}}_{a b}$. The MoncriefRinne evolution equations are second-order in spatial derivatives of the metric and much more complicated than our evolution equations, but there are fewer of them, both because our system is first-order and because the spatial triad vectors have three more degrees of freedom than the conformal metric. Since Moncrief and Rinne also impose the CMC hypersurface condition, their equation for the lapse is essentially the same as ours, and there is the same breakdown in smoothness due to polyhomogeneous terms. Their conformal factor is defined differently by demanding that the conformal scalar curvature be uniform in both space and time, but it satisfies a similar elliptic equation. The difference in conformal gauge does not affect the leading behavior of the conformal factor at $\dot{J}^{+}$, and the basic structure of the apparently singular terms in the equations is the same. Since the triad angular velocity plays no role in a coordinate-based system, Moncrief and Rinne have three fewer elliptic equations than we do. Their variable $\Gamma$ defined in their Eq. (2.11) is equivalent to our $\tilde{\alpha} \tilde{K}$, but is determined by a quite different elliptic equation. They note the advantages of imposing a boundary condition on $\Gamma$ equivalent to our Eq. (A31). Their elliptic equation for the shift preserves the spatial harmonic condition on the spatial coordinates.

The trade-off between complicated spatially secondorder evolution equations and more, but simpler, first-order evolution equations will probably make little difference in computational efficiency; however, high accuracy may be easier to achieve with our first-order equations. The more serious numerical efficiency issue in our formalism is the three extra elliptic equations. All of our variables except for the triad vectors and the shift vector are spatial coordinate scalars, which with the globally optimized triad orientations provided by the Nester gauge, may be better behaved in complicated dynamic scenarios than the coordinate components of the metric tensor and the conformal extrinsic curvature. In both approaches, it is critical to use highly optimized elliptic solvers.

Our asymptotic wave amplitude is $\hat{\tilde{\kappa}}_{a b} \doteq \hat{\tilde{K}}_{a b}$, whose coordinate components are $\breve{\chi}_{A B}$ in the asymptotic expansion of the spatial metric in Gaussian normal coordinates developed in Appendix A. To see how this relates to the Bondi news, we solve asymptotically for the coordinate transformation between our Gaussian normal coordinates defined on CMC hypersurfaces and Bondi coordinates. The inverse metric in Bondi coordinates $u, x, \bar{x}^{A^{\prime}}$, with $u$ the retarded time, $x$ the inverse of the Bondi radius $r$, and $\bar{x}^{A^{\prime}}$ the angular coordinates, is

$$
\begin{aligned}
\bar{g}^{u u} & =\bar{g}^{u A^{\prime}}=0, \quad \bar{g}^{u x}=x^{2} e^{-2 \beta}, \quad \bar{g}^{x x}=x^{4} W e^{-2 \beta}, \\
\bar{g}^{x A^{\prime}} & =x^{2} U^{A^{\prime}} e^{-2 \beta}, \quad \bar{g}^{A^{\prime} B^{\prime}}=x^{2} \bar{h}^{A^{\prime} B^{\prime}},
\end{aligned}
$$

with the determinant of $\bar{h}^{A^{\prime} B^{\prime}}$ equal to $(\sin \theta)^{-2}$. For a certain class of solutions of the Einstein equations, the Bondi-Sachs metric functions $\beta, W, U^{A^{\prime}}$, and $\bar{h}^{A^{\prime} B^{\prime}}$ have expansions in powers of $x$ away from future null infinity at $x=0$, provided that the Penrose regularity condition is satisfied (see [29]).

For the special class of inertial Bondi coordinates (see Sec. 6 of Ref. [6] and Ref. [8]), the angular metric $\bar{h}_{A^{\prime} B^{\prime}}$ has the expansion

$$
\bar{h}_{A^{\prime} B^{\prime}}=\breve{h}_{A^{\prime} B^{\prime}}+\bar{\chi}_{A^{\prime} B^{\prime}} x+O\left(x^{3}\right),
$$

where as in Appendix A, $\breve{h}_{A^{\prime} B^{\prime}}$ is the unit sphere metric, $\bar{\chi}_{A^{\prime} B^{\prime}}$ is a traceless, symmetric tensor on the unit sphere, and the absence of an $O\left(x^{2}\right)$ term is equivalent to the Penrose regularity condition in this context. Also, $\beta \doteq 0$ and $U^{A^{\prime}} \doteq 0$. The Bondi mass aspect $M$, whose average over the solid angle is the Bondi energy, is defined by $W=$ $1-2 M\left(u, \bar{x}^{A^{\prime}}\right) x+O\left(x^{2}\right)$. The Bondi news is basically the time derivative of $\bar{\chi}_{A^{\prime} B^{\prime}}$, often represented as a complex scalar $c$ whose real part is $\bar{\chi}^{\theta^{\prime}}{ }_{\theta^{\prime}}=-\bar{\chi}^{\varphi^{\prime}}{ }_{\varphi^{\prime}}$ and imaginary part is $\bar{\chi}^{\varphi^{\prime}}{ }_{\theta^{\prime}} \sin \theta^{\prime}=\bar{\chi}^{\theta^{\prime}}{ }_{\varphi^{\prime}} / \sin \theta^{\prime}$. The square of the Bondi news at a given physical point on $\mathcal{J}^{+}$is invariant under transformations of the Bondi coordinates from one inertial Bondi frame to another [4].

In Appendix A, we discuss the asymptotic conformal spatial metric in Gaussian normal coordinates $z, x^{A}$ and obtain asymptotic expressions for the lapse and shift which preserve the CMC hypersurface and Gaussian normal coordinate conditions. While these spatial coordinates are not the computational coordinates, the shift satisfies the same boundary condition at $\mathcal{J}^{+}$. The boundary condition on the 
conformal lapse, as discussed in Sec. VII, ensures that the coordinate time $t$ on the CMC hypersurfaces asymptotically corresponds to Minkowski retarded time. The coordinate transformation from these coordinates to the Bondi-Sachs coordinates can be constructed as a power series in the distance $z$ from $\mathcal{J}^{+}$, starting from $u \doteq t$ and $\bar{x}^{A^{\prime}} \doteq x^{A}$, by requiring that the transformed metric have the Bondi-Sachs form, with $\bar{g}^{u u}=\bar{g}^{u A^{\prime}}=0$. The result is

$$
u=t+\frac{z}{2 \tilde{\alpha}_{0}}+\frac{\tilde{\kappa}_{0}}{8 \tilde{\alpha}_{0}} z^{2}+O\left(z^{3}\right), \quad \bar{x}^{A^{\prime}}=x^{A}+O\left(z^{3}\right) .
$$

Then

$$
x^{2} \bar{h}^{A^{\prime} B^{\prime}}=\Omega^{2} \tilde{h}^{C D} \frac{\partial \bar{x}^{A^{\prime}}}{\partial x^{C}} \frac{\partial \bar{x}^{B^{\prime}}}{\partial x^{D}}+O\left(z^{6}\right),
$$

and equating the determinants of the two sides gives

$$
x=\Omega \xi\left(1+O\left(z^{3}\right)\right)=\frac{K \xi_{0}}{3} z+O\left(z^{2}\right) .
$$

Note that $\partial x / \partial t=O\left(z^{2}\right)$, since our boundary conditions keep $\partial \xi / \partial t \doteq 0$. From the expansions of $\bar{h}^{A^{\prime} B^{\prime}}$ and $\tilde{h}^{C D}$ (Eq. (A5)), we see that

$$
\bar{\chi}_{A^{\prime} B^{\prime}} \doteq-\frac{6}{K \xi_{0}} \breve{\chi}_{A B} .
$$

The expansion of $\mathcal{J}^{+}$clearly vanishes. From

$$
x^{2} e^{-2 \beta}=\Omega^{2} \frac{\beta^{z}}{\tilde{\alpha}^{2}} \frac{\partial x}{\partial z} \frac{\partial u}{\partial t}+O\left(z^{4}\right),
$$

$\beta=O(z)$, and from

$$
x^{2} U^{A^{\prime}} e^{-2 \beta}=\Omega^{2}\left(-\frac{\beta^{B} \beta^{z}}{\tilde{\alpha}^{2}} \frac{\partial x}{\partial z} \frac{\partial \bar{x}^{A^{\prime}}}{\partial x^{B}}+O\left(z^{3}\right)\right),
$$

$U^{A^{\prime}}=O\left(z^{2}\right)$. Therefore, all of the conditions for an inertial Bondi frame are satisfied, and the Bondi-Sachs "news" is $\dot{\bar{\chi}}_{A^{\prime} B^{\prime}} \equiv \partial_{u} \bar{\chi}_{A^{\prime} B^{\prime}}$. The vacuum Einstein equations in BondiSachs coordinates give, since $\partial_{u} \doteq \partial_{t}$,

$$
\begin{aligned}
& \frac{\partial M}{\partial u}=-\frac{1}{8} \breve{h}^{A^{\prime} C^{\prime}} \breve{h}^{B^{\prime} D^{\prime}} \dot{\bar{\chi}}_{A^{\prime} B^{\prime}} \dot{\bar{\chi}}_{C^{\prime} D^{\prime}}+\frac{1}{4} \dot{\bar{\chi}}^{A^{\prime} B^{\prime}}{ }_{\breve{\mid} A^{\prime} B^{\prime}} \\
& =-\frac{1}{2}\left[\left(\frac{3}{K \xi_{0}}\right)^{2} \breve{h}^{A C} \breve{h}^{B D} \dot{\check{\chi}}_{A B} \dot{\check{\chi}}_{C D}+\left(\frac{3}{K \xi_{0}}\right) \dot{\check{\chi}}^{A B} \breve{\mid ~} A B\right] \text {, }
\end{aligned}
$$

with $\breve{\mid}$ denoting covariant derivatives on the unit sphere [47]. The monopole and dipole parts of the term linear in $\breve{\chi}^{A B}$ as integrated over the unit sphere vanish identically, so this term contributes to neither the Bondi energy nor the Bondi momentum. Equation (80) defines the precise physical meaning of $\breve{\chi}_{A B}$ as the 2-tensor describing the asymptotic amplitude of the gravitational waves. This 2-tensor is equivalent to the 3-tensor whose triad components are $\tilde{\kappa}_{a b} \doteq \hat{\tilde{K}}_{a b}$, as projected into $\dot{J}^{+}$.

Our extraction of the Bondi news is actually quite a bit simpler than it is in Cauchy-characteristic matching or
Cauchy-characteristic extraction formalisms [6]. In the characteristic methods, the matching or extraction is done on a world tube in the interior, and the constraints controlling the conformal Bondi frame are integrated outward on the null hypersurface, generically resulting in a noninertial Bondi frame at $\mathcal{J}^{+}$. The subsequent transformation from the computational frame to the inertial Bondi frame requires considerable numerical effort [6], but a more direct way of calculating the Bondi news from an arbitrary characteristic frame is presented and tested in Ref. [8].

Although we contend that the approach we have presented holds much promise for high accuracy calculations of gravitational waveforms from 3D numerical simulations of the Einstein equations, it remains to be seen whether or not a stable and efficient numerical implementation can be achieved. A good place to begin is to fine-tune the numerical solution of the elliptic equations of our method in the context of the initial value problem and then evaluate whether or not the computation time required is prohibitive. Further analysis of the well-posedness of the entire hyperbolic-elliptic system would provide a theoretical foundation for a more extensive numerical effort.

\section{ACKNOWLEDGMENTS}

We thank Frank Estabrook, Luis Lehner, Anil Zenginoğlu, and particularly Vincent Moncrief for a number of discussions and helpful suggestions. O.S. was supported in part by grants CONACyT 61173 and CIC 4.19 to Universidad Michoacana. L. B. held a Visitor in Physics appointment at the California Institute of Technology during the course of this work and is grateful to Mark Scheel and Kip Thorne for their hospitality.

\section{APPENDIX A: ASYMPTOTIC EXPANSIONS IN GAUSSIAN NORMAL COORDINATES}

Quite a bit can be learned about the asymptotic behavior of the conformal factor and the regularization of the apparently singular terms in the evolution equations at $\mathcal{J}^{+}$by developing an explicit asymptotic solution. This is awkward to do in our implementation of the tetrad formalism because in the elliptic equations of the Nester gauge, there is no local control over how the orientation of the spatial triad vectors and therefore how $\hat{\tilde{N}}_{a b}$ evolves in a neighborhood of $\dot{J}^{+}$. On the other hand, by working directly with the coordinate components of the spatial metric and other tensors in the $\mathrm{CMC}$ hypersurfaces, and by making a special choice of spatial coordinates (Gaussian normal coordinates based on $\dot{J}^{+}$as a two-surface embedded in the 3D conformal space [27]) in a neighborhood of $\mathcal{J}^{+}$, we can straightforwardly obtain explicit asymptotic solutions for the conformal factor and extrinsic curvature in terms of quantities defined from the asymptotic expansion of the conformal spatial metric as a power series in the conformal 
proper distance from $\dot{J}^{+}$. Moncrief and Rinne [13] have done a similar asymptotic analysis in a more general coordinate system, but at the cost of substantial additional complexity.

The Gaussian normal coordinates are constructed by starting from angular coordinates on the $\dot{\mathcal{J}}^{+}$2-surface, which can always be chosen so that its metric is conformal to the standard unit sphere metric, with coordinates $x^{A}=(\theta, \phi)$. These angular coordinates are propagated inward from $\mathcal{J}^{+}$along normal spacelike geodesics in the CMC hypersurface, and a "radial" coordinate $z$ is defined to be equal to the proper distance from $\dot{\mathcal{J}}^{+}$along these geodesics. This construction gives a conformal spatial metric of the form

$$
d \tilde{s}^{2}=d z^{2}+\tilde{h}_{A B} d x^{A} d x^{B},
$$

since the geodesics normal to the $\dot{J}^{+} 2$-surface are perpendicular to the 2-surfaces of constant proper distance. Furthermore, since any metric on a 2-surface which has the topology of a two-sphere is conformal to the standard unit sphere metric $\breve{h}_{A B}$, we can decompose

$$
\tilde{h}_{A B}=\xi^{-2} \breve{h}_{A B},
$$

defining a $2 \mathrm{D}$ conformal factor $\xi$ such that

$$
\tilde{h}=\operatorname{det} \tilde{h}_{A B}=\xi^{-4} \operatorname{det} \breve{h}_{A B}=\xi^{-4} \sin ^{2} \theta .
$$

Assuming a power series expansion of the 2D conformal metric about $\mathcal{J}^{+}$through order $z^{2}$, we then have the form

$\tilde{h}_{A B}=\xi^{-2}\left[\breve{h}_{A B}-2 \breve{\chi}_{A B} z+\left(\breve{\chi}^{C D} \breve{\chi}_{C D} \breve{h}_{A B}-\breve{\psi}_{A B}\right) z^{2}+O\left(z^{3}\right)\right]$.

Here $\breve{\chi}_{A B}$ and $\breve{\psi}_{A B}$ are traceless, symmetric tensors with respect to the unit sphere metric $\breve{h}_{A B}$. The inverse is

$\tilde{h}^{A B}=\xi^{2}\left[\breve{h}^{A B}+2 \breve{\chi}^{A B} z+\left(\breve{\chi}^{C D} \breve{\chi}_{C D} \breve{h}^{A B}+\breve{\psi}^{A B}\right) z^{2}+O\left(z^{3}\right)\right]$.

It has been argued $([27,29])$ that in a general treatment of radiation in asymptotically flat spacetimes, there should be polyhomogeneous terms in this expansion, i.e., $z \log z$ and/ or $z^{2} \log z$ and higher order terms. Such terms are consistent with the zero-shear condition, for instance, and do not prevent the existence of $\mathcal{J}^{+}$as a null surface in the conformal space-time. As noted in Sec. I, we see no reason why such terms should be present when dealing with isolated systems in an astrophysical context. There may be polyhomogeneous terms present at higher orders in the expansion of $\tilde{h}_{A B}$ arising from the polyhomogeneous terms in the expansion of the extrinsic curvature that are associated with the CMC gauge choice.

Physical interpretation of the asymptotic solutions is greatly simplified if the 3D conformal gauge is such that 2 -surfaces of constant conformal radius correspond to 2 -spheres of the asymptotic flat space-time approaching
$\dot{\mathcal{J}}^{+}$on a CMC hypersurface. Since the level surfaces of the 3D conformal factor $\Omega$ approach $\dot{J}^{+}$uniformly in the conformal radius coordinate, this is accomplished by demanding that the intrinsic geometry of $\dot{J}^{+}$be that of a 2 -sphere, i.e., that the $2 \mathrm{D}$ conformal factor $\xi$ be a constant $\xi_{0}$, independent of angle, on $\dot{J}^{+}$, or equivalently, that the 2D scalar curvature of $\dot{\mathcal{J}}^{+},{ }^{2} R \doteq 2 \xi_{0}{ }^{2}$, be uniform. Assuming that the initial data satisfy this condition, it will be preserved if $\partial_{t} \xi \doteq 0$. As a result of our asymptotic analysis, we shall see this requires a certain Dirichlet boundary condition on the trace $\tilde{K}$ of the $3 \mathrm{D}$ conformal extrinsic curvature. In the context of our elliptic equations for preserving the Nester triad gauge, this provides the natural boundary condition on $\tilde{\alpha} \tilde{K}=-(3 / 2) \tilde{D}_{a} \zeta_{a}$. The same point was noted in the different context of Ref. [13].

The Gaussian normal coordinates are not suitable as global coordinates, since they break down somewhere in the interior as the normal spacelike geodesic congruence develops caustics, but they are guaranteed to be valid in a neighborhood of $\dot{\mathcal{J}}^{+}$, assuming a regular conformal geometry. Any breakdown of smoothness at $\mathcal{J}^{+}$cannot be attributed to the Gaussian normal choice of spatial coordinates.

The nonzero Christoffel symbols for the metric of Eq. (A1) are just

$$
\begin{aligned}
& \tilde{\Gamma}_{B z}^{A}=\frac{1}{2} \tilde{h}^{A C} \partial_{z} \tilde{h}_{C B}=-\tilde{\kappa}_{B}^{A}, \\
& \tilde{\Gamma}_{A B}^{z}=-\frac{1}{2} \partial_{z} \tilde{h}_{A B}=\tilde{\kappa}_{A B}, \quad \tilde{\Gamma}_{B C}^{A}={ }^{2} \tilde{\Gamma}_{B C}^{A},
\end{aligned}
$$

where $\tilde{\kappa}_{A B}$ are the coordinate components of the extrinsic curvature of the constant- $z$ 2-surfaces based on the outward normal, which is in the minus $z$-direction, and where ${ }^{2} \tilde{\Gamma}^{A}{ }_{B C}$ are the Christoffel symbols associated with the 2-metric $\tilde{h}_{A B}$. Let $\hat{\tilde{\kappa}}_{A B}$ denote the traceless part of $\tilde{\kappa}_{A B}$ and $\tilde{\kappa}$ the trace. Calculating the conformal 3D Ricci tensor, we get

$$
\begin{aligned}
\tilde{R}_{z}^{z} & =\partial_{z} \tilde{\kappa}-\tilde{\kappa}_{A B} \tilde{\kappa}^{A B}=\partial_{z} \tilde{\kappa}-\hat{\tilde{\kappa}}_{A B} \hat{\tilde{\kappa}}^{A B}-\frac{1}{2} \tilde{\kappa}^{2}, \\
\tilde{R}_{A}^{z} & =\tilde{\kappa}_{\mid A}-\tilde{\kappa}_{A \mid C}^{C}=\frac{1}{2} \tilde{\kappa}_{\mid A}-\hat{\tilde{\kappa}}^{C}{ }_{A \mid C}, \\
\tilde{R}_{B}^{A} & ={ }^{2} \tilde{R}_{B}^{A}+\partial_{z} \tilde{\kappa}_{B}^{A}-\tilde{\kappa} \tilde{\kappa}_{B}^{A} \\
& =\partial_{z} \hat{\tilde{\kappa}}_{B}^{A}-\tilde{\kappa} \hat{\tilde{\kappa}}_{B}^{A}+\frac{1}{2}\left({ }^{2} \tilde{R}+\partial_{z} \tilde{\kappa}-\tilde{\kappa}^{2}\right) \delta_{B}^{A} .
\end{aligned}
$$

The | symbol indicates a covariant derivative in the constant- $z$ 2-space with respect to the metric $\tilde{h}_{A B}$. The scalar curvature is

$$
\tilde{R}={ }^{2} \tilde{R}+2 \partial_{z} \tilde{\kappa}-\frac{3}{2} \tilde{\kappa}^{2}-\hat{\tilde{\kappa}}_{A B} \hat{\tilde{\kappa}}^{A B} .
$$

In terms of our expansion (A4) of the angular metric, it is easy to verify that

$$
\hat{\tilde{\kappa}}_{B}^{A}=\breve{\chi}_{B}^{A}+\breve{\psi}_{B}^{A} z+O\left(z^{2}\right),
$$


so $\hat{\tilde{\kappa}}_{B}^{A} \doteq \breve{\chi}_{B}^{A}$ and $\partial_{z} \hat{\tilde{\kappa}}_{B}^{A} \doteq \breve{\psi}^{A}{ }_{B}$. Also, $\tilde{\kappa}=(2 / \xi) \partial_{z} \xi$. Furthermore, assuming $\xi \doteq \xi_{0}$ is uniform on $\mathcal{J}^{+}$,

$$
\begin{aligned}
{ }^{2} \tilde{\Gamma}_{B C}^{A}= & \breve{\Gamma}_{B C}^{A}-\left[2 \breve{\chi}_{(B \backslash C)}^{A}-\breve{\chi}_{B C} \breve{l A}^{A}+\delta_{(B}^{A} \partial_{C)} \tilde{\kappa}_{0}\right. \\
& \left.-\frac{1}{2} \breve{h}_{B C} \breve{h}^{A D} \partial_{D} \tilde{\kappa}_{0}\right] z+O\left(z^{2}\right)
\end{aligned}
$$

and

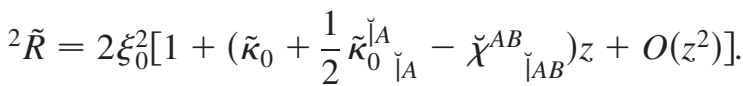

The $\mid$ symbol denotes a covariant derivative with respect to the unit sphere metric.

Our definition of the conformal factor is not quite the same as in Ref. [13] or [27], but like them, we can solve for the conformal factor to third order in the expansion away from $\dot{J}^{+}$. Write the expansion of $\Omega$ as

$$
\Omega=\frac{K}{3} z\left[1+c_{1}\left(x^{A}\right) z+c_{2}\left(x^{A}\right) z^{2}+O\left(z^{3}\right)\right],
$$

the expansion of $\tilde{\kappa}$ as

$$
\tilde{\kappa}=\tilde{\kappa}_{0}+\tilde{\kappa}_{1} z+O\left(z^{2}\right),
$$

and substitute into the Hamiltonian constraint, Eq. (38). The result is

$$
\begin{aligned}
\Omega= & \frac{K}{3} z\left\{1-\frac{1}{4} \tilde{\kappa}_{0} z+\frac{1}{12}\left[\frac{1}{2} \tilde{\kappa}_{0}^{2}-2 \tilde{\kappa}_{1}\right.\right. \\
& \left.\left.-\left(\hat{\tilde{K}}^{a b} \hat{\tilde{K}}_{a b}+\hat{\tilde{\kappa}}_{A B} \hat{\tilde{\kappa}}^{A B}-{ }^{2} \tilde{R}\right)_{\mathcal{J}^{+}}\right] z^{2}+O\left(z^{3}\right)\right\} .
\end{aligned}
$$

The expansion of $\Omega$ becomes locally indeterminate, and generically requires a polyhomogeneous $z^{3} \log z$ term, at the next order.

In the Gaussian normal coordinates, the outward normal to the constant $-z$-surfaces is just $\tilde{s}^{z}=-1, \quad \tilde{s}^{A}=0$. Denote the traceless part of the $3 \mathrm{D}$ extrinsic curvature as projected into these surfaces by

$$
\Sigma_{B}^{A} \equiv \hat{\tilde{K}}_{B}^{A}-\frac{1}{2} \delta^{A}{ }_{B} \hat{\tilde{K}}_{C}^{C} .
$$

The zero-shear condition derived in Sec. VI requires $\Sigma_{B}^{A} \doteq \hat{\tilde{\kappa}}_{B}^{A} \doteq \breve{\chi}_{B}^{A}$. Note that $\breve{\chi}^{A}{ }_{B}$ can be considered the asymptotic amplitude of the gravitational waves, and the zero-shear condition is a reflection of the fact that a derivative of the spatial metric along the normal to an asymptotically null hypersurface becomes equivalent to a radial derivative of the spatial metric as the normal becomes tangent to the hypersurface.

The momentum constraint Eq. (45) allows us to determine the leading asymptotic behavior of the rest of the extrinsic curvature in terms of $\breve{\chi}^{A}{ }_{B}$. In the Gaussian normal coordinate basis, we have

$$
\Omega \tilde{\nabla}_{j}\left(\Omega^{-1} \hat{\tilde{K}}_{i}^{j}\right)=\Omega^{-1} \partial_{j} \Omega \hat{\tilde{K}}_{i}^{j}-8 \pi G \Omega^{2} \tilde{j}_{i} .
$$

First consider the $z$-component, which through first-order in $z$ is

$$
\begin{gathered}
\frac{K}{3} z\left[\partial_{z}\left(\Omega^{-1} \hat{\tilde{K}}_{z}^{z}\right)+\left(\Omega^{-1} \hat{\tilde{K}}_{z}^{A}\right)_{\mid A}\right]+\hat{\tilde{\kappa}}^{B}{ }_{A} \Sigma_{B}^{A}{ }_{B} \\
-\frac{3}{2} \tilde{\kappa} \hat{\tilde{K}}_{z}^{z}=\frac{K}{3}\left(1-\frac{1}{2} \tilde{\kappa} z\right) \Omega^{-1} \hat{\tilde{K}}_{z}^{z} .
\end{gathered}
$$

Using the zero-shear condition, the zeroth-order result is

$$
\frac{K}{3 \Omega} \hat{\tilde{K}}_{z}^{z}=\frac{K}{3 \Omega} \tilde{s}^{a} \tilde{s}^{b} \hat{\tilde{K}}_{a b} \doteq \hat{\tilde{\kappa}}_{B}^{A} \Sigma^{B}{ }_{A} \doteq \breve{\chi}^{A}{ }_{B} \breve{\chi}^{B}{ }_{A} .
$$

The transverse components of the momentum constraint to first-order in $z$ are

$$
\begin{aligned}
& \frac{K}{3} z \partial_{z}\left(\Omega^{-1} \hat{\tilde{K}}_{A}^{z}\right)+\hat{\tilde{K}}_{A \mid B}^{B}-\tilde{\kappa} \hat{\tilde{K}}_{A}^{z} \\
& =\frac{K}{3 \Omega}\left(1-\frac{1}{2} \tilde{\kappa} z\right) \hat{\tilde{K}}_{A}^{z}-\frac{z}{2} \tilde{\kappa}_{\mid B} \hat{\tilde{K}}_{A}^{B} .
\end{aligned}
$$

Expand $\hat{\tilde{K}}_{A \mid B}^{B}=\Sigma^{B}{ }_{A \mid B}-\frac{1}{2}\left(\hat{\tilde{K}}_{z}^{z}\right)_{\mid A}$, using Eqs. (A8) and (A14), to get

$$
\begin{gathered}
\frac{K}{3} z \partial_{z}\left(\Omega^{-1} \hat{\tilde{K}}_{A}^{z}\right)+\breve{\chi}_{A \mid B}^{B}+z\left(\partial_{z} \Sigma^{B}{ }_{A}\right)_{\mid B} \\
-\frac{1}{2} \tilde{\kappa} \hat{\tilde{K}}_{A}-\frac{1}{2} \tilde{\kappa}_{\mid B} \breve{\chi}^{B}{ }_{A}=\frac{K}{3 \Omega} \hat{\tilde{K}}_{A}^{z} .
\end{gathered}
$$

At zeroth-order,

$$
\frac{K}{3 \Omega} \hat{\tilde{K}}_{A}^{z} \doteq \breve{\chi}_{A \breve{B}}^{B} .
$$

Using these zeroth-order results, we can calculate the first-order corrections. However, the equations cannot be satisfied by a simple power series expansion. Instead, let

$$
\frac{K}{3 \Omega} \hat{\tilde{K}}_{z}^{z}=\breve{\chi}_{B}^{A} \breve{\chi}_{A}^{B}+d_{1} z+d_{1}^{\prime} z \log z+O\left(z^{2}\right) .
$$

Upon substitution into Eq. (A13), the $d_{1}$ 's cancel, leaving $d_{1}$ indeterminate locally, but

$d_{1}^{\prime}=-\xi_{0}^{2} \breve{\chi}^{A B}{ }_{\breve{A} A B}-\breve{\chi}_{B}^{A}\left[\breve{\psi}^{B}{ }_{A}+\left(\partial_{z} \Sigma^{B}{ }_{A}\right)_{\mathcal{J}^{+}}-\tilde{\kappa}_{0} \breve{\chi}^{B}{ }_{A}\right]$.

Similarly, the coefficient of $z$ in the expansion of $(K / 3 \Omega) \hat{\tilde{K}}_{A}^{z}$ is indeterminate locally, and one has an expansion of the form

$$
\frac{K}{3 \Omega} \hat{\tilde{K}}_{A}^{z}=\breve{\chi}_{A \mid B}^{B}+e_{A} z+e_{A}^{\prime} z \log z+O\left(z^{2}\right),
$$

where the coefficient of $z \log z$ is

$$
e_{A}^{\prime}=-\left(\partial_{z} \Sigma_{A}^{B}-\frac{1}{2} \tilde{\kappa}_{0} \breve{\chi}^{B}{ }_{A}\right)_{\mid B} .
$$

It is also possible to calculate the coefficient $c_{3}^{\prime}$ of the $z^{3} \log z$ term in the expansion for the conformal factor. The result is $c_{3}^{\prime}=-d_{1}^{\prime} / 8$.

The expansions above allow us, using L'Hôpital's rule, to directly evaluate at $\dot{J}^{+}$the coordinate components of the apparently singular terms in the evolution equation for $\hat{\tilde{K}}_{a b}$, 


$$
S_{i j} \equiv \frac{1}{\Omega}\left(\tilde{\nabla}_{i} \tilde{\nabla}_{j} \Omega+\frac{K}{3} \hat{\tilde{K}}_{i j}\right)^{T F} .
$$

The result is

$$
\begin{aligned}
S_{z}^{z} \doteq & \frac{1}{3}\left[\breve{\chi}^{C}{ }_{D} \breve{\chi}^{D}{ }_{C}+2 \xi_{0}^{2}-\tilde{\kappa}_{1}\right], \\
S_{B}^{A} \doteq & \partial_{z}\left(\Sigma_{B}^{A}{ }_{B}\right)-\breve{\psi}^{A}{ }_{B}+\frac{1}{2} \tilde{\kappa}_{0} \breve{\chi}_{B}^{A} \\
& -\frac{1}{6}\left(\breve{\chi}^{C}{ }_{D} \breve{\chi}^{D}{ }_{C}+2 \xi_{0}^{2}-\tilde{\kappa}_{1}\right) \delta^{A}{ }_{B}, \\
S_{A}^{z} \doteq & \breve{\chi}_{A \backslash C}^{C}-\frac{1}{2} \partial_{A} \tilde{\kappa}_{0} .
\end{aligned}
$$

Finally, we compute the expansion of the conformal lapse $\tilde{\alpha}$ from the CMC slicing condition, Eq. (46):

$$
\begin{aligned}
\tilde{\alpha}= & \tilde{\alpha}_{0}\left[1-\frac{1}{2} \tilde{\kappa}_{0} z+\frac{1}{4}\left(\frac{1}{2} \tilde{\kappa}_{0}^{2}-\tilde{\kappa}_{1}-3 \breve{\chi}^{C}{ }_{D} \breve{\chi}^{D}{ }_{C}+2 \xi_{0}^{2}\right) z^{2}\right. \\
& \left.+O\left(z^{3}\right)\right] .
\end{aligned}
$$

In deriving this expansion, we have assumed that $\tilde{\alpha}_{0}$ is uniform as stipulated in Sec. VII.

\section{The Penrose regularity condition}

The apparently singular terms $S_{i j}$ are also related to the electric part of the physical Weyl tensor evaluated at $\mathcal{J}^{+}$. The Penrose regularity condition asserts that the Weyl tensor of the conformal space-time should vanish at $\mathcal{J}^{+}$. The conformal invariance of the Weyl tensor implies that the coordinate components of the electric tensor are invariant,

$$
E_{i j}=C_{i \mu j \nu} \lambda_{0}^{\mu} \lambda_{0}^{\nu}=\tilde{C}_{i \mu j \nu} \tilde{\lambda}_{0}^{\mu} \tilde{\lambda}_{0}^{\nu}=\tilde{E}_{i j} .
$$

A well-known expression for the physical Weyl electric tensor in a vacuum space-time (see Ref. [13]) is

$$
E_{i j}=R_{i j}-K_{i}{ }^{k} K_{k j}+K K_{i j},
$$

where the vacuum evolution equations have been used to eliminate time derivatives. Our assumptions about the falloff of the energy-momentum tensor at $\mathcal{J}^{+}$imply that Eq. (A23) holds there. In terms of the conformal quantities, the traceless part of this gives

$$
\begin{aligned}
\hat{\tilde{E}}_{i j} & =\left\{R_{i j}-\hat{K}_{i}{ }^{k} \hat{K}_{k j}\right\}^{T F}+\frac{K}{3} \hat{K}_{i j} \\
& =\frac{1}{\Omega}\left\{\tilde{\nabla}_{i} \tilde{\nabla}_{j} \Omega+\frac{K}{3} \hat{\tilde{K}}_{i j}\right\}^{T F}+\left\{\hat{\tilde{R}}_{i j}-\hat{\tilde{K}}_{i}{ }^{k} \hat{\tilde{K}}_{k j}\right\}^{T F} .
\end{aligned}
$$

Raising an index with the conformal metric in the Gaussian normal coordinates, applying the results in Eqs. (A6), (A7), and (A21), and noting the identity $\breve{\chi}^{A}{ }_{C} \breve{\chi}^{C}{ }_{B}=\frac{1}{2} \breve{\chi}^{C}{ }_{D} \breve{\chi}^{D}{ }_{C} \delta^{A}{ }_{B}$,

$$
\begin{aligned}
& \hat{\tilde{E}}_{z}^{z} \doteq \frac{1}{3}\left[\breve{\chi}^{C}{ }_{D} \breve{\chi}^{D}{ }_{C}+2 \xi_{0}^{2}-\tilde{\kappa}_{1}\right] \\
& +\frac{1}{3}\left[\tilde{\kappa}_{1}-2 \xi_{0}^{2}-2 \breve{\chi}^{C}{ }_{D} \breve{\chi}^{D}{ }_{C}\right] \\
& +\frac{1}{3} \breve{\chi}^{C}{ }_{D} \breve{\chi}^{D} \doteq 0 \\
& \hat{\tilde{E}}_{B}^{A} \doteq \partial_{z}\left(\Sigma^{A}{ }_{B}\right)-\breve{\psi}^{A}{ }_{B}+\frac{1}{2} \tilde{\kappa}_{0} \breve{\chi}^{A}{ }_{B} \\
& -\frac{1}{6}\left(\breve{\chi}^{C}{ }_{D} \breve{\chi}^{D}{ }_{C}+2 \xi_{0}^{2}-\tilde{\kappa}_{1}\right) \delta_{B}^{A}+\breve{\psi}_{B}^{A}-\tilde{\kappa}_{0} \breve{\chi}^{A}{ }_{B} \\
& +\frac{1}{6}\left(2 \xi_{0}^{2}+2 \breve{\chi}^{C}{ }_{D} \breve{\chi}^{D}{ }_{C}-\tilde{\kappa}_{1}\right) \delta_{B}^{A}-\frac{1}{6} \breve{\chi}^{C}{ }_{D} \breve{\chi}^{D}{ }_{C} \\
& \doteq \partial_{z}\left(\Sigma_{B}^{A}\right)-\frac{1}{2} \tilde{\kappa}_{0} \breve{\chi}_{B}^{A} \text {, }
\end{aligned}
$$

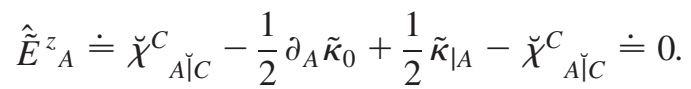

The Penrose regularity condition is not satisfied automatically once the zero-shear condition is satisfied; it requires the additional condition that

$$
\partial_{z}\left(\Sigma_{B}^{A}\right) \doteq \frac{1}{2} \tilde{\kappa}_{0} \breve{\chi}_{B}^{A} .
$$

In the Bondi-Sachs asymptotic expansion on null hypersurfaces, the Penrose regularity condition is the vanishing of the traceless part of the $r^{-2}$ term in the expansion of the metric of the two-surfaces of constant Bondi radius $r$ (see [29]). In that context the condition is preserved by the Einstein equations, once imposed in the initial data, and therefore the same must be true for Eq. (A27).

The magnetic part of the Weyl tensor is

$$
B_{i j}=\tilde{B}_{i j}=\tilde{\nabla}_{k} \hat{\tilde{K}}_{\ell(i} \tilde{\varepsilon}_{j)}{ }^{k \ell}
$$

and using the asymptotic expansions above, one finds

$$
\begin{aligned}
& B^{z}{ }_{z} \doteq 0, \quad B_{A}^{z} \doteq 0, \\
& B_{B}^{A}{ }_{B} \doteq-\breve{\varepsilon}_{C}^{A}\left[\partial_{z}\left(\Sigma_{B}^{C}\right)-\frac{1}{2} \tilde{\kappa}_{0} \breve{\chi}_{B}^{C}\right],
\end{aligned}
$$

with $B_{B}^{A} \doteq 0$ if and only if Eq. (A27) holds. Therefore, the magnetic part of the Weyl tensor does not give additional conditions.

Given that the Weyl tensor vanishes at $\mathcal{J}^{+}$, much simpler expressions can be obtained for the apparently singular terms in the evolution equations than those of Eq. (A21) directly in terms of tetrad quantities in a general coordinate system, without the need to propagate the normal geodesics away from $\dot{\mathcal{J}}^{+}$to calculate $\tilde{\kappa}_{a b}$, etc. One just uses the vanishing of the tetrad components of the electric part of the Weyl tensor. The regular form of the evolution equation at $\mathcal{J}^{+}$for $\hat{\tilde{K}}_{a b}$ is given in Eq. (74). 


\section{Metric evolution equations}

It is instructive to examine the asymptotic behavior of the evolution equations for the conformal spatial metric in terms of the conformal extrinsic curvature assuming the initial Gaussian normal coordinate condition is preserved. The general equations are

$$
\partial_{t} \tilde{h}_{i j}=\beta^{k} \partial_{k} \tilde{h}_{i j}+\tilde{h}_{k j} \partial_{i} \beta^{k}+\tilde{h}_{i k} \partial_{j} \beta^{k}+2 \tilde{\alpha}\left(\hat{\tilde{K}}_{i j}+\frac{1}{3} \tilde{h}_{i j} \tilde{K}\right) .
$$

The evolution equation for $\tilde{h}_{z z}$ becomes an equation for $\beta^{z}$,

$$
\partial_{z} \beta^{z}=-\tilde{\alpha}\left(\hat{\tilde{K}}_{z z}+\frac{1}{3} \tilde{K}\right)
$$

from which

$$
\beta^{z}=\tilde{\alpha}_{0}-\left(\frac{\tilde{\alpha} \tilde{K}}{3}\right)_{0} z-\frac{1}{2}\left[\tilde{\alpha}_{0} \breve{\chi}_{D}^{C} \breve{\chi}^{D}{ }_{C}+\left(\frac{\tilde{\alpha} \tilde{K}}{3}\right)_{1}\right] z^{2}+O\left(z^{3}\right) .
$$

The condition for keeping $\tilde{h}_{A z}=0$ is

$$
\partial_{z} \beta^{A}+\tilde{h}^{A C} \partial_{C} \beta^{z}=-2 \tilde{\alpha} \hat{\tilde{K}}_{z}^{A},
$$

from which

$$
\beta^{A}=\xi_{0}^{2}\left[\breve{h}^{A B} \partial_{B}\left(\frac{\tilde{\alpha} \tilde{K}}{6}\right)_{0}-\tilde{\alpha}_{0} \breve{\chi}_{\breve{~}{ }^{A B}}\right] z^{2}+O\left(z^{3}\right) .
$$

The boundary conditions on the shift and the lapse are from Sec. VII.

Decompose the evolution equations for the angular part of the metric as given by Eq. (A4) into trace and traceless parts. Defining the evolution of $\xi$ so Eq. (A3) is preserved, the trace in zeroth order gives

$$
\frac{1}{\xi} \partial_{t} \xi \doteq \frac{\beta^{z}}{\xi} \partial_{z} \xi-\frac{1}{3} \tilde{\alpha} \tilde{K} \doteq \tilde{\alpha}_{0}\left(\frac{1}{2} \tilde{\kappa}_{0}-\frac{1}{3} \tilde{K}\right),
$$

using $\tilde{\kappa}=2 \partial_{z} \log \xi$. The traceless part in zeroth order, given the zero-shear condition, confirms that $\partial_{t} \breve{h}_{A B} \doteq 0$, as assumed.

The condition for keeping the intrinsic geometry of $\mathcal{J}^{+}$ a true sphere, i.e., keeping $\xi$ uniform on $\mathcal{J}^{+}$, is $\partial_{t} \xi \doteq 0$. From Eq. (A30), we see this requires the boundary condition on $\tilde{K}$ to be

$$
\frac{2 \tilde{\alpha} \tilde{K}}{3} \doteq \tilde{\alpha} \tilde{\kappa}
$$

which can be implemented in the elliptic Nester gauge equations of Sec. VA. A significant consequence of this condition is that $\tilde{\alpha}-\beta^{z}=O\left(z^{2}\right)$.

At first order in $z$, and using the Gaussian-normalcoordinate shift, one finds from the trace

$$
\begin{aligned}
\tilde{D}_{0} \tilde{\kappa} & \doteq \frac{1}{\tilde{\alpha}_{0}}\left(\partial_{t} \tilde{\kappa}_{0}-\tilde{\alpha}_{0} \tilde{\kappa}_{1}\right) \\
& \doteq-\frac{1}{2} \tilde{\kappa}_{0}^{2}+\breve{\chi}^{C D} \breve{\chi}_{C D}-\frac{2}{3 \tilde{\alpha}_{0}} \partial_{z}(\tilde{\alpha} \tilde{K})
\end{aligned}
$$

and from the traceless part

$$
\partial_{t} \breve{\chi}_{B}^{A} \doteq \tilde{\alpha}_{0}\left(\breve{\psi}_{B}^{A}-\partial_{z} \Sigma_{B}^{A}\right) .
$$

After imposing the Penrose regularity condition, Eq. (A27),

$$
\partial_{t} \breve{\chi}_{B}^{A} \doteq \tilde{\alpha}_{0}\left(\breve{\psi}^{A}{ }_{B}-\frac{1}{2} \tilde{\kappa}_{0} \breve{\chi}^{A}{ }_{B}\right) .
$$

Next, we examine the evolution equation for the conformally rescaled extrinsic curvature,

$$
\begin{aligned}
& \partial_{t} \hat{\tilde{K}}_{j}^{i}=\beta^{k} \partial_{k} \hat{\tilde{K}}_{j}^{i}-\hat{\tilde{K}}_{j}^{k} \partial_{k} \beta^{i}+\hat{\tilde{K}}_{k}^{i} \partial_{j} \beta^{k} \\
& \quad+\tilde{\alpha}\left(-\tilde{R}_{j}^{i}+\frac{1}{\tilde{\alpha}} \tilde{\nabla}^{i} \tilde{\nabla}_{j} \tilde{\alpha}-\frac{\tilde{K}}{3} \hat{\tilde{K}}_{j}^{i}-2 S_{j}^{i}+8 \pi G \Omega^{3} \tilde{\sigma}_{j}^{i}\right)^{T F} .
\end{aligned}
$$

Evaluating the right-hand side of this equation at $\mathcal{J}^{+}$we obtain, using Eqs. (A6), (A14), (A16), (A21), (A22), (A28), and (A31),

$$
\begin{aligned}
\partial_{t} \hat{\tilde{K}}_{z}^{z} & \doteq 0, \quad \partial_{t} \hat{\tilde{K}}_{A}^{z} \doteq 0, \\
\partial_{t} \hat{\tilde{K}}_{B}^{A} & \doteq \tilde{\alpha}_{0}\left(\breve{\psi}_{B}^{A}{ }_{B}-\partial_{z} \Sigma_{B}^{A}\right) .
\end{aligned}
$$

In view of the Eq. (A33), this shows that the regularity conditions $\hat{\tilde{K}}_{z}^{z} \doteq 0, \hat{\tilde{K}}_{A}^{z} \doteq 0, \hat{\tilde{K}}_{B}^{A} \doteq \hat{\tilde{\kappa}}_{B}^{A}$ are automatically preserved in the time evolution.

Now return to the question of the apparent breakdown in smoothness at $\mathcal{J}^{+}$in the asymptotic expansion of the extrinsic curvature. Note that from Eq. (A20), the condition for the coefficient $e_{1}^{\prime}$ of the leading $\log$ term in $\hat{\tilde{K}}_{A}^{z}$ to vanish is identical to the Penrose regularity condition, Eq. (A27). However, applying the Penrose regularity condition to Eq. (A18) for the coefficient $d_{1}^{\prime}$ of the leading log term in $\hat{\tilde{K}}_{z}^{z}$ leaves

$$
\begin{aligned}
& d_{1}^{\prime}=-\xi_{0}^{2} \breve{\chi}^{A B}{ }_{\breve{A} A B}-\breve{\chi}^{A}{ }_{B}\left[\breve{\psi}^{B}{ }_{A}-\frac{1}{2} \tilde{\kappa}_{0} \breve{\chi}^{B}{ }_{A}\right] \\
& =-\xi_{0}^{2} \breve{\chi}^{A B}{ }_{\breve{A} A}-\frac{1}{2 \tilde{\alpha}} \partial_{t}\left(\breve{\chi}^{A}{ }_{B} \breve{\chi}^{B}{ }_{A}\right),
\end{aligned}
$$

which does not vanish if any outgoing waves are present at $\mathcal{J}^{+}$. Therefore, the lack of smoothness of $\hat{\tilde{K}}_{z}^{z}$ at $\mathcal{J}^{+}$ cannot be prevented by a regularity condition and must be dealt with in any calculation involving emission of gravitational radiation. Friedrich's proofs of smoothness at $\mathcal{J}^{+}$given smooth initial data $[9,48]$ do not apply because they are based on solving a complete symmetric hyperbolic system of evolution equations. Our assumption of CMC hypersurfaces requires an elliptic equation for the lapse, and it is this elliptic equation which generates a breakdown in smoothness in the presence of outgoing radiation. The elliptic equations which enforce the Nester gauge are not relevant because they do not affect the evolution of the coordinate metric. Our mathematical results on the presence of polyhomogeneous terms in the asymptotic expansion of the extrinsic curvature and conformal factor, after sorting through differences in notation, 
are equivalent to those of Sec. 4 of Andersson and Chruściel [27].

We do not expect the lack of smoothness at $\mathcal{J}^{+}$to be a serious impediment to the accuracy and/or stability of numerical calculations, even though in principle it could prevent rapid convergence of spectral-based numerical methods.

\section{APPENDIX B: CONSTRAINT PROPAGATION}

In this appendix, we derive the constraint propagation system for our evolution scheme. This system describes the evolution of constraint violations which could be present in the initial data or, more generally, could be triggered by truncation errors in a numerical scheme.

Since the constraint Eq. (43) is required for vanishing torsion, we should allow for a connection $\nabla$ with nonzero torsion tensor $T$ in the constraint propagation system. An efficient method for deriving this system [49] is based on the Riemann and Bianchi identities for the curvature tensor $R$,

$$
\begin{gathered}
\sum_{(\beta \gamma \delta)} R_{\beta \gamma \delta}^{\alpha}=\sum_{(\beta \gamma \delta)}\left(\nabla_{\beta} T_{\gamma \delta}^{\alpha}+T^{\alpha}{ }_{\epsilon \delta} T_{\beta \gamma}^{\epsilon}\right), \\
\sum_{(\beta \gamma \delta)} \nabla_{\beta} R^{\alpha}{ }_{\epsilon \gamma \delta}=-\sum_{(\beta \gamma \delta)} R_{\epsilon \kappa \delta}^{\alpha} T_{\beta \gamma}^{\kappa},
\end{gathered}
$$

where $\sum_{(\beta \gamma \delta)}$ denotes the cyclic sum over $(\beta \gamma \delta)$. Expressed in terms of an arbitrary basis $\mathbf{e}_{\alpha}$ of vector fields, the torsion and curvature tensors are defined by

$$
\begin{aligned}
T_{\gamma \delta}^{\alpha} \equiv & -\left[\mathbf{e}_{\gamma}, \mathbf{e}_{\delta}\right]^{\alpha}-2 \Gamma_{[\gamma \delta]}^{\alpha}, \\
R_{\beta \gamma \delta}^{\alpha} \equiv & e_{\gamma}\left(\Gamma_{\beta \delta}^{\alpha}{ }_{\beta \delta}\right)-e_{\delta}\left(\Gamma_{\beta \gamma}^{\alpha}\right)+\Gamma_{\beta \delta}^{\epsilon} \Gamma^{\alpha}{ }_{\epsilon \gamma} \\
& -\Gamma_{\beta \gamma}^{\epsilon} \Gamma_{\epsilon \delta}^{\alpha}-\Gamma_{\beta \epsilon}^{\alpha}\left[\mathbf{e}_{\gamma}, \mathbf{e}_{\delta}\right]^{\epsilon},
\end{aligned}
$$

respectively. If the frame $\mathbf{e}_{\alpha}$ is orthonormal, Einstein's field equations are equivalent to the requirements that the connection coefficients $\Gamma_{\alpha \beta \gamma}=\Gamma_{[\alpha \beta] \gamma}$ are antisymmetric in $\alpha \beta$, that the torsion tensor is zero, and that the Ricci tensor $R_{\alpha \beta} \equiv R_{\alpha \gamma \beta}^{\gamma}$ is related to the stress-energy tensor $\tau_{\alpha \beta}$ according to $R_{\alpha \beta}=8 \pi G \tau_{\alpha \beta}-4 \pi G \eta_{\alpha \beta} \eta^{\gamma \delta} \tau_{\gamma \delta}$. An equivalent way of stating this is the satisfaction of the equations

$$
T_{\gamma \delta}^{\alpha}=0, \quad \Delta_{\alpha \beta \gamma \delta}=0,
$$

where

$\Delta_{\alpha \beta \gamma \delta} \equiv R_{\alpha \beta \gamma \delta}-\Gamma_{\alpha \beta \epsilon} T_{\gamma \delta}^{\epsilon}-C_{\alpha \beta \gamma \delta}-S_{\alpha \beta \gamma \delta}$,

$S_{\alpha \beta \gamma \delta} \equiv 8 \pi G\left(\eta_{\alpha[\gamma} \tau_{\delta] \beta}-\eta_{\beta[\gamma} \tau_{\delta] \alpha}-\frac{2}{3} \eta_{\alpha[\gamma} \eta_{\delta] \beta} \eta^{\epsilon \kappa} \tau_{\epsilon \kappa}\right)$,

and $C_{\alpha \beta \gamma \delta}$ is a tensor field satisfying the algebraic symmetries $\quad C_{[\alpha \beta \gamma] \delta}=0, \quad C_{[\alpha \beta][\gamma \delta]}=C_{\alpha \beta \gamma \delta}=C_{\gamma \delta \alpha \beta}$, $\eta^{\alpha \gamma} C_{\alpha \beta \gamma \delta}=0$. For a solution of the field Eqs. (B3), $C_{\alpha \beta \gamma \delta}$ is the Weyl curvature tensor associated with the geometry.
In order to relate Eq. (B3) to our evolution and constraint equations, we assume that the timelike leg $\mathbf{e}_{0}=\mathbf{n}$ coincides with the future-directed unit normal to the time slices $\Sigma_{t}$. This implies that $T^{0}{ }_{\gamma \delta}=0$. We decompose the remaining components of $T$ and $\Delta$ according to

$$
\begin{aligned}
T_{\alpha \gamma \delta}= & 2 F_{\alpha[\gamma} n_{\delta]}+G_{\alpha \beta} \varepsilon^{\beta}{ }_{\gamma \delta}, \\
\Delta_{\alpha \beta \gamma \delta}= & -4 n_{[\alpha} \mathcal{E}_{\beta][\gamma} n_{\delta]}-\varepsilon_{\alpha \beta}{ }^{{ }^{\prime}} \mathcal{D}_{\iota \kappa} \varepsilon^{\kappa}{ }_{\gamma \delta} \\
& -2 n_{[\alpha} \mathcal{B}_{\beta]_{\iota}} \varepsilon^{\iota}{ }_{\gamma \delta}+2 \varepsilon_{\alpha \beta}{ }^{\iota} \mathcal{H}_{\iota[\gamma \gamma} n_{\delta]},
\end{aligned}
$$

where $\varepsilon_{\beta \gamma \delta} \equiv n^{\alpha} \varepsilon_{\alpha \beta \gamma \delta}$ and the covariant tensor fields $\mathbf{F}$, $\mathbf{G}, \mathcal{E}, \mathcal{D}, \mathcal{B}$ and $\mathcal{H}$ are orthogonal to $\mathbf{n}$. The left and right duals of $\Delta$ are defined by

$$
\begin{aligned}
& (* \Delta)_{\alpha \beta \gamma \delta} \equiv \frac{1}{2} \varepsilon_{\alpha \beta}{ }^{\epsilon \kappa} \Delta_{\epsilon \kappa \gamma \delta}, \\
& (\Delta *)_{\alpha \beta \gamma \delta} \equiv \frac{1}{2} \Delta_{\alpha \beta \epsilon \kappa} \varepsilon_{\gamma \delta}^{\epsilon \kappa},
\end{aligned}
$$

respectively, and induce the following transformations in the decomposition of $\Delta$ :

$\Delta \mapsto * \Delta: \mathcal{E} \mapsto \mathcal{H}, \quad \mathcal{D} \mapsto \mathcal{B}, \quad \mathcal{B} \mapsto-\mathcal{D}, \quad \mathcal{H} \mapsto-\mathcal{E}$,

$\Delta \mapsto \Delta *: \mathcal{E} \mapsto \mathcal{B}, \quad \mathcal{D} \mapsto \mathcal{H}, \quad \mathcal{B} \mapsto-\mathcal{E}, \quad \mathcal{H} \mapsto-\mathcal{D}$.

The trace of $\Delta$ is given by

$$
\begin{aligned}
\Delta_{\alpha \gamma \beta}^{\gamma}= & R_{\alpha \beta}+\Gamma_{\alpha}{ }^{\gamma \delta} T_{\delta \gamma \beta}-8 \pi G\left(\tau_{\alpha \beta}-\frac{1}{2} \eta_{\alpha \beta} \eta^{\gamma \delta} \tau_{\gamma \delta}\right) \\
= & n_{\alpha} n_{\beta} \mathcal{E}^{\gamma}{ }_{\gamma}-n_{\alpha} \varepsilon_{\beta}{ }^{\gamma \delta} \mathcal{B}_{\gamma \delta}+\varepsilon_{\alpha}{ }^{\gamma \delta} \mathcal{H}_{\gamma \delta} n_{\beta}-\mathcal{E}_{\alpha \beta} \\
& +\mathcal{D}_{\beta \alpha}-\left(\eta_{\alpha \beta}+n_{\alpha} n_{\beta}\right) \mathcal{D}^{\gamma}{ }_{\gamma} .
\end{aligned}
$$

The Weyl tensor has a similar decomposition to that of $\Delta$. However, its algebraic symmetries imply that $\mathcal{D}=\mathcal{E}$, $\mathcal{H}=\mathcal{B}$, and that $\mathcal{E}$ and $\mathcal{B}$ are symmetric and traceless.

Our evolution system is obtained from the combinations of the field Eqs. (B3) which eliminate the Weyl tensor. More precisely, the evolution Eqs. (14), (9), and (10) are

$0=F_{a b} B_{a}{ }^{k}=-D_{0} B_{b}{ }^{k}+$ l.o.,

$0=\mu_{a b} \equiv \mathcal{E}_{a b}-\mathcal{D}_{a b}=-D_{0} K_{b a}+\varepsilon_{b c d} D_{c} N_{d a}+$ l.o.,

$0=\nu_{a b} \equiv \mathcal{B}_{a b}-\mathcal{H}_{a b}=-D_{0} N_{b a}-\varepsilon_{b c d} D_{c} K_{d a}+$ l.o.,

where "l.o." stands for lower order terms which do not contain derivatives of $B_{a}{ }^{k}, K_{a b}, N_{a b}, a_{b}, \omega_{b}$ or $\tau_{\alpha \beta}$. The constraint Eqs. (15) and (11)-(13), are equivalent to

$$
\begin{aligned}
& 0=G_{a b} B_{a}{ }^{k}=-\varepsilon_{b c d} D_{c} B_{d}{ }^{k}+\text { l.o., } \\
& 0=M \equiv-\mathcal{D}_{a a}=2 D_{a} n_{a}+\text { l.o., } \\
& 0=P_{a} \equiv \varepsilon_{a b c} \mathcal{B}_{b c}=D_{b} K_{a b}-D_{a} K+\text { l.o., } \\
& 0=Q_{a} \equiv \varepsilon_{a b c} \mathcal{D}_{b c}=D_{b} N_{a b}-D_{a} N+\text { l.o., }
\end{aligned}
$$


and the equation $\mathcal{B}_{a a}=0$ is automatically satisfied as a consequence of the hypersurface orthogonal gauge.

Now we use the identities (B1) and (B2) to derive evolution equations for the constraint variables $G_{a b}, M$, $P_{a}$ and $Q_{a}$. For this, we first rewrite them in terms of $T$ and $\Delta$. Using the algebraic symmetries of $C_{\alpha \beta \gamma \delta}$ and the fact that $\sum_{(\beta \gamma \delta)} S_{\alpha \beta \gamma \delta}=0$, this yields

$$
\sum_{(\beta \gamma \delta)} \nabla_{\beta} T_{\alpha \gamma \delta}=\sum_{(\beta \gamma \delta)}\left[\Delta_{\alpha \beta \gamma \delta}+\left(\mathbf{e}_{\alpha} \cdot\left[\mathbf{e}_{\epsilon}, \mathbf{e}_{\beta}\right]+\Gamma_{\alpha \epsilon \beta}\right) T_{\gamma \delta}^{\epsilon}\right],
$$

$$
\begin{aligned}
& \sum_{(\beta \gamma \delta)} \nabla_{\beta}\left(\Delta_{\alpha \epsilon \gamma \delta}+C_{\alpha \epsilon \gamma \delta}+S_{\alpha \epsilon \gamma \delta}\right) \\
& =\sum_{(\beta \gamma \delta)}\left[-\Gamma_{\alpha \epsilon \kappa} \Delta_{\beta \gamma \delta}{ }_{\beta \gamma}-\mathbf{e}_{k}\left(\Gamma_{\alpha \epsilon \beta}\right) T_{\gamma \delta}^{\kappa}\right] .
\end{aligned}
$$

We may further simplify these equations by noticing that

$$
\begin{aligned}
\sum_{(\beta \gamma \delta)} \nabla_{\beta} S_{\alpha \epsilon \gamma \delta} & =\frac{1}{2} \varepsilon_{\beta \gamma \delta \iota} \varepsilon^{\iota \beta^{\prime} \gamma^{\prime} \delta^{\prime}} \nabla_{\beta^{\prime}} S_{\alpha \epsilon \gamma^{\prime} \delta^{\prime}} \\
& =\varepsilon_{\beta \gamma \delta}{ }^{\iota} \nabla^{\kappa}(S *)_{\alpha \epsilon \iota \kappa}, \\
\sum_{(\beta \gamma \delta)} \Delta^{\kappa}{ }_{\beta \gamma \delta} & =\varepsilon_{\beta \gamma \delta \alpha}(\Delta *)^{\kappa \epsilon \alpha}{ }_{\epsilon},
\end{aligned}
$$

where $\quad(S *)_{\alpha \beta \gamma \delta}=8 \pi G\left(\varepsilon_{\gamma \delta[\alpha}{ }^{\kappa} \tau_{\beta] \kappa}-\varepsilon_{\alpha \beta \gamma \delta} \eta^{\iota \kappa} \tau_{\iota \kappa} / 3\right)$. Using the expression (B6) for the trace of $\Delta$ and the transformation properties (B5) of the right dual, we find

$$
\begin{array}{ll}
(\Delta *)^{0 \epsilon}{ }_{0 \epsilon}=0, & (\Delta *)^{0 \epsilon} \epsilon_{b \epsilon}=Q_{b}+\varepsilon_{b c d} \mu_{c d}, \\
(\Delta *)_{a \epsilon 0}^{\epsilon}=Q_{a}, & (\Delta *)_{a \epsilon b}=-\varepsilon_{a b c} P_{c}-\nu_{b a}+\delta_{a b} \nu_{c c} .
\end{array}
$$

As a consequence, the right-hand sides of Eqs. (B7) and (B8) are a linear combination of the constraint fields $G_{a b}$, $P_{a}, Q_{a}$ and the evolution variables $F_{a b}, \mu_{a b}, \nu_{a b}$.

Equation (B7) with $F_{a b}=0$ yields the following identities:

$$
\begin{gathered}
\varepsilon_{b c d} \mu_{c d}+Q_{b}-a_{a} G_{a b}=0, \\
{\left[\left(D_{b}-2 n_{b}\right) G_{a b}\right] D_{a}=Q_{a} D_{a}+G_{a b}\left[D_{a}, D_{b}\right],} \\
D_{0} G_{a b}=-\varepsilon_{a b c} P_{c}-\nu_{b a}+\delta_{a b} \nu_{c c}+K_{a c} G_{c b}+G_{a c} K_{c b} \\
-K G_{a b}+\varepsilon_{a c d} G_{c b} \omega_{d}+G_{a c} \varepsilon_{b c d} \omega_{d} .
\end{gathered}
$$

The first identity is an algebraic relation between the antisymmetric part of the evolution equation $\mu_{a b}=0$ and the constraint fields $Q_{a}$ and $G_{a b}$. It can also be obtained directly by computing the antisymmetric part of Eq. (9). Upon using the commutator relation $\varepsilon_{b c d} D_{c} D_{d}=$ $-\left(G_{a b}+N_{a b}-\delta_{a b} N\right) D_{a}$, the second identity yields a differential relation between the constraint fields $Q_{a}$ and $G_{a b}$. The third identity gives an evolution equation for $G_{a b}$.

In order to obtain evolution equations for the remaining constraint fields $M, P_{a}$ and $Q_{a}$, we contract Eq. (B8) and its left dual over $\alpha \beta$ and then over $\delta \epsilon$. Assuming that the stress-energy tensor is divergence-free, this yields the twice contracted Bianchi identities

$$
\begin{aligned}
& \nabla_{\alpha} \Delta_{\gamma \beta}^{\gamma \alpha}-\frac{1}{2} \nabla_{\beta} \Delta_{\gamma \delta}^{\gamma \delta} \\
& =* \Gamma_{\beta}{ }_{\gamma \delta}(\Delta *)^{\epsilon}{ }_{\delta \epsilon \gamma}-\mathbf{e}_{\delta}\left(\Gamma^{\alpha \gamma}\right) T_{\alpha \beta}^{\delta}+\frac{1}{2} \mathbf{e}_{\delta}\left(\Gamma_{\beta}^{\alpha \gamma}\right) T_{\alpha \gamma}^{\delta},
\end{aligned}
$$

$$
\begin{aligned}
& \nabla_{\alpha}(* \Delta)_{\gamma \beta}^{\gamma \alpha}-\frac{1}{2} \nabla_{\beta}(* \Delta)_{\gamma \delta}^{\gamma \delta} \\
& =-\Gamma_{\beta}{ }^{\gamma \delta}(\Delta *)^{\epsilon}{ }_{\delta \epsilon}-\mathbf{e}_{\delta}\left(* \Gamma_{\gamma}^{\alpha \gamma}\right) T_{\alpha \beta}^{\delta}+\frac{1}{2} \mathbf{e}_{\delta}\left(* \Gamma_{\beta}^{\alpha \gamma}\right) T_{\alpha \gamma}^{\delta},
\end{aligned}
$$

where $* \Gamma_{\alpha \beta \kappa} \equiv \varepsilon_{\alpha \beta \gamma \delta} \Gamma^{\gamma \delta}{ }_{\kappa} / 2$. Using the expression (B6) for the trace of $\Delta$ and its left dual, we find, in terms of the constraint and evolution variables,

$$
\begin{aligned}
\Delta^{\gamma 0}{ }_{\gamma 0} & =M-\mu_{a a}, \\
\Delta^{\gamma 0}{ }_{\gamma b} & =-P_{b}, \\
\Delta^{\gamma}{ }_{a \gamma 0} & =-P_{a}+\varepsilon_{a c d} \nu_{c d}, \\
\Delta^{\gamma}{ }_{a \gamma b} & =-\varepsilon_{a b c} Q_{c}+\delta_{a b} M-\mu_{a b}, \\
(* \Delta)^{\gamma 0}{ }_{\gamma 0} & =\nu_{a a}, \\
(* \Delta)_{\gamma b}^{\gamma 0} & =Q_{b}, \\
(* \Delta)^{\gamma}{ }_{a \gamma 0} & =Q_{a}+\varepsilon_{a c d} \mu_{c d}, \\
(* \Delta)_{a \gamma b}^{\gamma} & =-\varepsilon_{a b c} P_{c}+\nu_{a b} .
\end{aligned}
$$

By virtue of Eqs. (B9) and (B10) it turns out that the zero component of Eq. (B13) is satisfied identically, while Eq. (B12) and the spatial components of Eq. (B13) give

$$
\begin{gathered}
D_{0} M+D_{a} P_{a}=\varepsilon_{b c d} D_{b} \nu_{c d}+\text { l.o., } \\
D_{0} P_{b}-\varepsilon_{b c d} D_{c} Q_{d}+D_{b} M=-D_{a} \mu_{a b}+D_{b} \mu_{a a}+\text { l.o., } \\
D_{0} Q_{b}+\varepsilon_{b c d} D_{c} P_{d}=-D_{a} \nu_{a b}+D_{b} \nu_{a a}+\text { l.o. }
\end{gathered}
$$

The constraint propagation system for the original evolution system, Eqs. (14), (9), and (10), is given by Eqs. (B11) and (B14)-(B16) where the evolution equations $\mu_{a b}=$ $\nu_{a b}=0$ are imposed. The lower order terms "l.o." depend linearly on the constraint fields $G_{a b}, M, P_{b}$ and $Q_{b}$, but not on their derivatives. Therefore, the constraint propagation equations form a linear, first-order symmetric hyperbolic system, and it follows that the constraints are preserved by the evolution system (14), (9), and (10).

In the CMC and 3D Nester gauges, the evolution equations we impose are slightly different. In order to see this, we decompose 


$$
\begin{gathered}
\mu_{a b}=\hat{\mu}_{a b}+\varepsilon_{a b c} \mu_{c}+\frac{\delta_{a b}}{3} \mu, \\
\nu_{a b}=\hat{\nu}_{a b}+\varepsilon_{a b c} \nu_{c}+\frac{\delta_{a b}}{3} \nu,
\end{gathered}
$$

where $\hat{\mu}_{a b}$ and $\hat{\nu}_{a b}$ denote the symmetric, trace-free parts of $\mu_{a b}$ and $\nu_{a b}$, respectively. Then, the equations $\hat{\mu}_{a b}=$ $\hat{\nu}_{a b}=0$ yield the evolution Eqs. (16) and (17). Equation (18), which yields the elliptic equation for the lapse, is equivalent to $\mu=M$. However, when writing this equation in terms of the conformally rescaled fields, the Hamiltonian constraint is used again to eliminate the most singular terms at $\mathcal{J}^{+}$. This amounts to setting $\mu=0$. Next, Eqs. (19) and (20), which are used to derive the elliptic system for preserving the 3D Nester gauge, are obtained from $\nu=0$ and $2 \nu_{a}=P_{a}$, respectively. Using this together with $2 \mu_{b}=-Q_{b}+a_{a} G_{a b}$ and Eq. (B10), the constraint propagation system yields

$$
\begin{gathered}
D_{0} G_{a b}=\text { l.o. } \\
D_{0} M=\text { l.o. } \\
D_{0} P_{b}-\frac{1}{2} \varepsilon_{b c d} D_{c} \bar{Q}_{d}+D_{b} M=\text { l.o. } \\
D_{0} \bar{Q}_{b}+\frac{1}{2} \varepsilon_{b c d} D_{c} P_{d}=\text { l.o. }
\end{gathered}
$$

where $\bar{Q}_{b} \equiv Q_{b}+a_{a} G_{a b}$. This system is weakly hyperbolic. However, if the Hamiltonian constraint $M=0$ is imposed, then the constraint evolution system is described by the Eqs. (B17), (B19), and (B20) which yield a linear, first-order symmetric hyperbolic system with propagation speeds relative to normal observers equal to zero and one half the speed of light.

Depending on the election for the shift vector in Sec. V B, there might be additional constraints or small adjustments to be made in the constraint propagation system. For the spatial harmonic gauge, an additional constraint is $\tilde{V}^{k}=0$, whose propagation equation is of the form $\tilde{D}_{0} \tilde{V}^{k}=$ l.o. For the minimal conformal strain and distortion conditions (61) and (63), the triad vectors $\mathbf{B}_{a}$ are propagated along the time evolution vector field $\partial_{t}$ instead of $\tilde{D}_{0}$, see Eq. (59), which corresponds to setting the combination $\alpha F_{a b}+\varepsilon_{b c d} G_{a c} \beta_{d}$ to zero instead of $F_{a b}=0$.

\section{APPENDIX C: THE RELATION BETWEEN THE 3D NESTER GAUGE CONDITION AND THE 3D DIRAC EQUATION}

In this appendix, we first review the relation found in Ref. [42] between orthonormal three-frames satisfying the 3D Nester gauge and nowhere vanishing spinor fields satisfying the 3D Dirac equation. Then, we reconsider the equations in Sec. IV C which are responsible for preserving the 3D Nester gauge and our choice of conformal factor throughout evolution, and show that they can be rewritten as an inhomogeneous 3D Dirac equation. Based on these observations, we show how to recast these equations into second-order elliptic form.

The Dirac equation over a three-dimensional Riemannian manifold $(\Sigma, h)$ with triad field $\mathbf{B}_{a}$ and corresponding connection coefficients $\Gamma_{a b c}$ is

$$
i \sigma_{a}\left(D_{a}+\frac{1}{2} \Gamma_{c d a} \Sigma_{c d}\right) \xi=m \xi
$$

where $\xi: \Sigma \rightarrow \mathbb{C}^{2}$ is a $S U(2)$-spinor field on $\Sigma$,

$$
\sigma_{1} \equiv\left(\begin{array}{cc}
0 & 1 \\
1 & 0
\end{array}\right), \quad \sigma_{2} \equiv\left(\begin{array}{cc}
0 & -i \\
i & 0
\end{array}\right), \quad \sigma_{3} \equiv\left(\begin{array}{cc}
1 & 0 \\
0 & -1
\end{array}\right),
$$

are the Pauli spin matrices, $\Sigma_{c d} \equiv \frac{1}{4}\left[\sigma_{c}, \sigma_{d}\right]$ and $m$ is the mass of the field. Equation $(\mathrm{C} 1)$ is invariant with respect to local rotations

$$
\xi=U \bar{\xi}, \quad \mathbf{B}_{a}=R_{a b} \overline{\mathbf{B}}_{b}
$$

with $U: \Sigma \rightarrow S U(2)$ and $R: \Sigma \rightarrow S O(3)$ the corresponding rotation defined by $U \sigma_{a} U^{*}=\sigma_{b} R_{b a}$. In terms of the notation introduced in Sec. II, Eq. (C1) can be rewritten in the following way,

$$
i \sigma_{a}\left(D_{a}-n_{a}\right) \xi=\left(m+\frac{1}{2} N\right) \xi
$$

Now, suppose the frame $\mathbf{B}_{a}$ satisfies the integrated 3D Nester gauge conditions (30), such that $n_{a}=D_{a} \Phi$ and $N=$ const. Setting $m \equiv-N / 2$, Eq. (C3) reduces to $i \sigma_{a} D_{a}\left(e^{-\Phi} \xi\right)=0$ with the solution $\xi=e^{\Phi} \xi_{0}, \xi_{0} \in \mathbb{C}^{2}$ being a constant spinor. Conversely, suppose $\xi=\left(\xi^{0}, \xi^{1}\right)^{T}$ is a solution of Eq. (C1) which is nowhere vanishing. Then, the rotation

$$
U \equiv \frac{1}{p}\left(\begin{array}{cc}
\xi^{0} & -\left(\xi^{1}\right)^{*} \\
\xi^{1} & \left(\xi^{0}\right)^{*}
\end{array}\right), \quad p \equiv|\xi|,
$$

leads to the constant direction spinor solution $\bar{\xi}=p(1,0)^{T}$ of Eq. (C3). It follows that the new frame $\overline{\mathbf{B}}_{a}$ satisfies $\bar{n}_{a}=\bar{D}_{a}(\log p)$ and $\bar{N}=-2 m$, and hence the integrated 3D Nester gauge.

Therefore, the existence and uniqueness of a three-frame satisfying the integrated 3D Nester gauge condition (C3) with $N=N_{0}=$ const is equivalent to the existence and uniqueness of a nowhere vanishing spinor field satisfying the three-dimensional Dirac Eq. (C1) with mass $m=$ $-N_{0} / 2$. The latter being a linear, elliptic equation, it is amenable to an analysis using standard tools from elliptic theory. Existence and uniqueness theorems for similar equations have been obtained in Refs. [50,51] in the context of the positivity of the ADM and Bondi masses.

While the direction of the spinor $\xi$ is related to rotations of the triad, its norm is related to conformal transformations thereof. Indeed, the Dirac Eq. (C1) is invariant with respect to the conformal transformation $\xi=\Omega \tilde{\xi}$, 
$\mathbf{B}_{a}=\Omega \tilde{\mathbf{B}}_{a}$, where $\Omega>0$ is a strictly positive function on $\Sigma$. This feature may be used in order to fix the conformal factor (up to a positive multiplicative constant) such that the rescaled quantities satisfy $\tilde{n}_{a}=0$. This corresponds to the choice for the conformal factor in Sec. V.

Finally, consider Eqs. (31) and (32) for the quantities $v_{0} \equiv 2 \alpha D_{0} \Phi$ and $v_{a} \equiv \alpha \omega_{a}$ which are responsible for preserving the $3 \mathrm{D}$ Nester gauge and the choice of the conformal factor throughout evolution. It turns out that these equations are equivalent to the inhomogeneous, 3D Dirac equation

$$
i \sigma_{a} D_{a} \xi=F, \quad F \equiv-\alpha\left(\begin{array}{c}
\gamma_{0}-i \gamma_{3} \\
\gamma_{2}-i \gamma_{1}
\end{array}\right)
$$

for the spinor

$$
\xi=\left(\begin{array}{c}
v_{0}-i v_{3} \\
v_{2}-i v_{1}
\end{array}\right)
$$

where $\quad \gamma_{0} \equiv \hat{N}_{a b} \hat{K}_{a b}+\frac{1}{3} K N+D_{0} N \quad$ and $\quad \gamma_{a} \equiv$ $-\varepsilon_{a b c} \hat{K}_{b d} \hat{N}_{d c}+\hat{K}_{a b}\left(3 n_{b}+a_{b}\right)-\frac{2}{3} K a_{a}-8 \pi G j_{a}$. This can be partially understood by considering the rotations defined in Eq. (C4) which map the orthonormal threeframe to one that satisfies the 3D Nester gauge. Parametrizing $U$ by its rotation angle $\Theta$ and axis $e_{a}$, $e_{a} e_{a}=1$, we have

$$
U=\exp \left(\frac{\Theta}{2 i} e_{a} \sigma_{a}\right)=\cos \left(\frac{\Theta}{2}\right)-i \sin \left(\frac{\Theta}{2}\right) e_{a} \sigma_{a} .
$$

Hence, the corresponding spinor field is

$$
\xi=p\left(\begin{array}{c}
\cos \left(\frac{\Theta}{2}\right)-i \sin \left(\frac{\Theta}{2}\right) e_{3} \\
\left(e_{2}-i e_{1}\right) \sin \left(\frac{\Theta}{2}\right)
\end{array}\right) .
$$

For an infinitesimal rotation and change $p=p_{0}+\delta p$ in the conformal factor, like the one required to preserve the $3 \mathrm{D}$ Nester gauge and our condition on the conformal factor in an infinitesimal time step, this yields precisely a spinor of the form (C6) with $v_{0} \equiv \delta p$ and $v_{a} \equiv p_{0} \delta \Theta e_{a} / 2$.

Equation (C5) constitutes an elliptic system of equations for the fields $v_{0}$ and $v_{b}$ which can be solved subject to appropriate boundary conditions. A second-order elliptic system can be obtained from this by the ansatz $\xi=$ $i \sigma_{b} D_{b} \eta$, with $\eta$ a potential spinor field. Introduced into Eq. (C5) this yields

$$
-D_{a} D_{a} \eta+i N_{a b} \sigma_{b} D_{a} \eta-i N \sigma_{a} D_{a} \eta=F,
$$

where we have used the commutation relation $\varepsilon_{c a b} D_{a} D_{b}=N_{b c} D_{b}-N D_{c}$. In terms of the potentials $\psi$ and $\zeta_{a}$ introduced in Sec. VA, the potential spinor is given by

$$
\eta=-\left(\begin{array}{l}
\psi-i \zeta_{3} \\
\zeta_{2}-i \zeta_{1}
\end{array}\right)
$$

[1] F. Pretorius, in Physics of Relativistic Objects in Compact Binaries: From Birth to Coalescence (Springer, New York, 2009), pp. 305-369.

[2] J. Centrella, J. G. Baker, B. J. Kelly, and J. R. van Meter, Rev. Mod. Phys. 82, 3069 (2010).

[3] H. Bondi, M. G. J. van der Burg, and A. W. K. Metzner, Proc. R. Soc. A 269, 21 (1962).

[4] R. K. Sachs, Proc. R. Soc. A 270, 103 (1962).

[5] J. M. Stewart, Proc. R. Soc. A 424, 211 (1989).

[6] J. Winicour, Living Rev. Relativity 12 (2009); http:// www.livingreviews.org/lrr-2009-3.

[7] C. Reisswig, N. T. Bishop, D. Pollney, and B. Szilágyi, Classical Quantum Gravity 27, 075014 (2010).

[8] M. C. Babiuc, B. Szilágyi, J. Winicour, and Y. Zlochower, arXiv:1011.4223v1.

[9] H. Friedrich, Commun. Math. Phys. 91, 445 (1983).

[10] J. Frauendiener, Living Rev. Relativity 7 (2004); http:// www.livingreviews.org/lrr-2004-1.

[11] H. Friedrich, Commun. Math. Phys. 103, 35 (1986).

[12] A. Zenginoğlu, Classical Quantum Gravity 25, 195025 (2008).

[13] V. Moncrief and O. Rinne, Classical Quantum Gravity 26, 125010 (2009).
[14] O. Rinne, Classical Quantum Gravity 27, 035014 (2010).

[15] A. Zenginoğlu and M. Tiglio, Phys. Rev. D 80, 024044 (2009).

[16] A. Zenginoğlu and L. E. Kidder, Phys. Rev. D 81, 124010 (2010).

[17] L. T. Buchman, H. P. Pfeiffer, and J. M. Bardeen, Phys. Rev. D 80, 084024 (2009).

[18] F.B. Estabrook and H.D. Wahlquist, Living Rev. Relativity 12, 46 (2009).

[19] L. T. Buchman and J.M. Bardeen, Phys. Rev. D 67, 084017 (2003); 72, 049903(E) (2005).

[20] F. B. Estabrook, R. S. Robinson, and H.D. Wahlquist, Classical Quantum Gravity 14, 1237 (1997).

[21] F. B. Estabrook, Phys. Rev. D 71, 044004 (2005).

[22] F.B. Estabrook, Classical Quantum Gravity 23, 2841 (2006).

[23] L. T. Buchman and J.M. Bardeen, Phys. Rev. D 72, 124014 (2005).

[24] H. Friedrich, Classical Quantum Gravity 13, 1451 (1996).

[25] J. M. Nester, J. Math. Phys. (N.Y.) 30, 624 (1989).

[26] J. M. Nester, Classical Quantum Gravity 8, L19 (1991).

[27] L. Andersson and P. T. Chruściel, Commun. Math. Phys. 161, 533 (1994). 
[28] R. Penrose, Proc. R. Soc. A 284, 159 (1965).

[29] P. T. Chruściel, M. A. H. MacCallum, and D. B. Singleton, Phil. Trans. Roy. Soc. Lond., A 350, 113 (1995).

[30] L. Andersson and V. Moncrief, Ann. Inst. Henri Poincaré 4, 1 (2003).

[31] S. Bonazzola, E. Gourgoulhon, P. Grandclément, and J. Novak, Phys. Rev. D 70, 104007 (2004).

[32] I. Cordero-Carrión, J. M. Ibáñez, E. Gourgoulhon, J. L. Jaramillo, and J. Novak, Phys. Rev. D 77, 084007 (2008).

[33] I. Cordero-Carrión, P. Cerdá-Durán, H. Dimmelmeier, J. L. Jaramillo, J. Novak, and E. Gourgoulhon, Phys. Rev. D 79, 024017 (2009).

[34] M. Holst, L. Lindblom, R. Owen, H. P. Pfeiffer, M. A. Scheel, and L. E. Kidder, Phys. Rev. D 70, 084017 (2004).

[35] http://www.black-holes.org/SpEC.html.

[36] H.P. Pfeiffer, L.E. Kidder, M.A. Scheel, and S. A. Teukolsky, Comput. Phys. Commun. 152, 253 (2003).

[37] R.M. Wald, General Relativity (The University of Chicago Press, Chicago, 1984).

[38] E. T. Newman and R. Penrose, J. Math. Phys. (N.Y.) 3, 566 (1962); 4, 998(E) (1963).

[39] C. W. Misner, K. S. Thorne, and J. A. Wheeler, Gravitation (W. H. Freeman and Company, San Francisco, 1973).
[40] N. Straumann, General Relativity and Relativistic Astrophysics (Springer Verlag, Berlin, 1984).

[41] J. M. Nester, J. Math. Phys. (N.Y.) 33, 910 (1992).

[42] A. Dimakis and F. Müller-Hoissen, Phys. Lett. A 142, 73 (1989).

[43] R. Penrose, in Relativity, Groups, and Topology (Gordon and Breach, New York, 1964), pp. 565-584.

[44] J. W. York, in Sources of Gravitational Radiation, edited by L. L. Smarr (Cambridge University Press, Cambridge, 1979), pp. 83-126.

[45] R. Arnowitt, S. Deser, and C. W. Misner, in Gravitation: An Introduction to Current Research, edited by L. Witten (Wiley, New York, 1962), pp. 227-265.

[46] L. Smarr and J.W. York, Phys. Rev. D 17, 2529 (1978).

[47] P. T. Chruściel, J. Jezierski, and M. A. H. MacCallum, Phys. Rev. D 58, 084001 (1998).

[48] H. Friedrich, Commun. Math. Phys. 107, 587 (1986).

[49] H. Friedrich and G. Nagy, Commun. Math. Phys. 201, 619 (1999).

[50] O. Reula, J. Math. Phys. (N.Y.) 23, 810 (1982).

[51] O. Reula and K.P. Tod, J. Math. Phys. (N.Y.) 25, 1004 (1984). 